\title{
WEIGHTED CALDERÓN-ZYGMUND ESTIMATES FOR WEAK SOLUTIONS OF QUASI-LINEAR DEGENERATE ELLIPTIC EQUATIONS
}

\author{
TUOC V. PHAN
}

\begin{abstract}
This paper studies the Sobolev regularity estimates for weak solutions of a class of degenerate, and singular quasi-linear elliptic problems of the form $\operatorname{div}[\mathbb{A}(x, u, \nabla u)]=\operatorname{div}[\mathbf{F}]$ with non-homogeneous Dirichlet boundary conditions over bounded non-smooth domains. The coefficients $\mathbb{A}$ could be be singular, and degenerate or both in $x$ in the sense that they behave like some weight function $\mu$, which is in the $A_{2}$ class of Muckenhoupt weights. Global and interior weighted $W^{1, p}(\Omega, \omega)$-regularity estimates are established for weak solutions of these equations with some other weight function $\omega$. The results obtained are even new for the case $\mu=1$ because of the dependence on the solution $u$ of $\mathbb{A}$. In case of linear equations, our $W^{1, p}$-regularity estimates can be viewed as the Sobolev's counterpart of the Hölder's regularity estimates established by B. Fabes, C. E. Kenig, and R. P. Serapioni.
\end{abstract}

Keywords: Degenerate quasi-linear elliptic equations, Muckenhoupt weights, Two weighted norm inequalities, Nonlinear weighted Calderón-Zygmund estimates.

\section{INTRODUCTION AND MAIN RESULTS}

Let $\Omega$ be a bounded domain in $\mathbb{R}^{n}$ with boundary $\partial \Omega$, and let $\mathbb{K}$ be an open interval in $\mathbb{R}$, or $\mathbb{K}=\mathbb{R}$. The theme of the paper is to establish the interior and global weighted $W^{1, p}$-regularity estimates for weak solutions of the non-homogeneous Dirichlet boundary value problem

$$
\left\{\begin{array}{cccc}
\operatorname{div}[\mathbb{A}(x, u, \nabla u)] & = & \operatorname{div}[\mathbf{F}(x)] & \text { in } \Omega, \\
u & = & g(x) & \text { on } \partial \Omega,
\end{array}\right.
$$

where $\mathbf{F}: \Omega \rightarrow \mathbb{R}^{n}$ is a given measurable vector field, $g: \Omega \rightarrow \mathbb{R}$ is a given measurable function. Moreover, the vector field function $\mathbb{A}: \Omega \times \mathbb{K} \times \mathbb{R}^{n} \rightarrow \mathbb{R}^{n}$ is a Carathéodory mapping satisfying the following natural coercivity, growth conditions

$$
\begin{aligned}
& \mathbb{A}(x, \cdot, \cdot) \text { is continuous on } \mathbb{K} \times \mathbb{R}^{n}, \text { for almost every } x \in \Omega, \\
& \mathbb{A}(\cdot, z, \eta) \text { is measurable for each fixed }(z, \eta) \in \mathbb{K} \times \mathbb{R}^{n}, \\
& |\mathbb{A}(x, z, \eta)| \leq \Lambda \mu(x)|\eta|, \quad \text { for almost every } x \in \Omega, \text { for all }(z, \eta) \in \mathbb{K} \times \mathbb{R}^{n}, \\
& \Lambda^{-1} \mu(x)|\xi|^{2} \leq\langle\mathbb{A}(x, z, \xi), \xi\rangle, \text { for almost every } x \in \Omega \text {, for all }(z, \xi) \in \mathbb{K} \times \mathbb{R}^{n},
\end{aligned}
$$

where $\Lambda>0$ is a fixed constant, and $\mu: \mathbb{R}^{n} \rightarrow[0, \infty]$ is a weight function in the Muckenhoupt class $A_{2}$.

We assume further that the vector field $\mathbb{A}$ is asymptotically Uhlenbeck in the following sense: There is a symmetric measurable matrix $\tilde{\mathbb{A}}: \Omega \rightarrow \mathbb{R}^{n \times n}$, and a bounded continuous function $\omega_{0}: \overline{\mathbb{K}} \times[0, \infty) \rightarrow[0, \infty)$ such that

$$
|\mathbb{A}(x, z, \eta)-\tilde{\mathbb{A}}(x) \eta| \leq \omega_{0}(z,|\eta|)[1+|\eta|] \mu(x), \quad \text { for all most every } \quad x \in \Omega, \quad \forall(z, \eta) \in \mathbb{K} \times \mathbb{R}^{n},
$$

and

$$
\lim _{s \rightarrow \infty} \omega_{0}(z, s)=0 \text {, uniformly in } z \text {, for } z \in \overline{\mathbb{K}} \text {. }
$$

Observe that from (1.4) and (1.5), we can assume also that

$$
\Lambda^{-1} \mu(x)|\xi|^{2} \leq\langle\tilde{\mathbb{A}}(x) \xi, \xi\rangle \leq \Lambda \mu(x)|\xi|^{2}, \quad \forall \xi \in \mathbb{R}^{n}, \quad \text { a.e. } \quad x \in \Omega .
$$

Our prototypical example is of the form

$$
\mathbb{A}(x, z, \eta)=a(x, z,|\eta|) \eta \text {, with } \lim _{s \rightarrow \infty} a(x, z, s)=\tilde{a}(x) \text {, uniformly in } z \in \overline{\mathbb{K}} \text {, and } \tilde{\mathbb{A}}(x)=\tilde{a}(x) \mathbb{I}_{n} .
$$


For a given weak solution $u \in W^{1,2}(\Omega, \mu)$ of $(1.1)$, the weighted Sobolev space with the weight $\mu$, the main objective is to investigate the validity of the following types of higher regularity weighted estimates

$$
\int_{\Omega}|\nabla u|^{p} \omega(x) d x \leq C\left[\int_{\Omega}|\mathbf{F} / \mu|^{p} \omega(x) d x+\int_{\Omega}|\nabla g|^{p} \omega(x) d x+\omega(\Omega)\left\{\left(\frac{1}{\mu(\Omega)} \int_{\Omega}|\nabla u|^{2} \mu(x) d x\right)^{p / 2}+1\right\}\right]
$$

with $p>2$, and some other weight function $\omega$ in some class of Muckenhoupt weights, whose definitions will be given later.

The purpose of this paper is twofold. On one hand, it is the continuation of the developments of the recent work [2, 17, 36, 37] on the theory of Sobolev regularity theory for weak solutions of quasi-linear elliptic equations in which the coefficients $\mathbb{A}$ are dependent on the solution $u$. See, for instance [1, 3, 5, 6, 7, 8, 9, 12, 13, 23, 24, 25, 26, 27, 28, 29, 21, 44] for other work in the same directions but only for linear equations or for equations in which $\mathbb{A}$ is independent on $u$. On the other hand, this work includes the case that $\mathbb{A}$ could be singular or degenerate as a weight in some Munkenhoupt class of weights as considered in many papers such as [15, 16, 22, 30, 31, 35, 38, 41, 42] in which only Schauder's regularity of weak solutions are investigated. Moreover, even for the uniformly elliptic case, the results in this paper also improve those in [2, 17, 36, 37] since they do not require a-priori boundedness of weak solutions of 1.1). This work also extends the recent work [8] to the nonlinear case and two weighted estimates. Results of this paper can be considered as the Sobolev's regularity counterpart of the Schauder's one established in [15, 16, 22, 30, 31, 35, 38, 41, 42] for singular, degenerate equations.

We need several notations and definitions before stating our results. For each weight function $\mu$ and $p \geq 1$, the weighted Lebesgue space $L^{p}(\Omega, \mu)$ is defined as

$$
L^{p}(\Omega, \mu)=\left\{f: \Omega \rightarrow \mathbb{R}:\|f\|_{L^{p}(\Omega, \mu)}^{p}=\int_{\Omega}|f(x)|^{p} \mu(x) d x<\infty\right\} .
$$

As in [15], if $\mu \in A_{2}$ and an open bounded domain $\Omega \subset \mathbb{R}^{n}$, we can define the weighted Sobolev space $W^{1,2}(\Omega, \mu)$ consisting of all measurable functions $f: \Omega \rightarrow \mathbb{R}$ such that $f \in L^{2}(\Omega, \mu)$ and all of their weak derivatives $\partial_{x_{k}} f \in L^{2}(\Omega, \mu)$ with $k=1,2, \cdots, n$. Moreover,

$$
\|f\|_{W^{1,2}(\Omega, \mu)}=\|f\|_{L^{2}(\Omega, \mu)}+\sum_{k=1}^{n}\left\|\partial_{x_{k}} f\right\|_{L^{2}(\Omega, \mu)} .
$$

We also denote $W_{0}^{1,2}(\Omega, \mu)$ the closure of the compactly supported function space $C_{0}^{\infty}(\Omega)$ in $W^{1,2}(\Omega, \mu)$. For convenience in stating the results, we need a notation of the class of vector fields satisfying (1.2)-(1.8).

Definition 1.1. Given an open set $\Omega \subset \mathbb{R}^{n}$, an open interval $\mathbb{K} \subset \mathbb{R}$, and the numbers $\Lambda>0, M_{0} \geq 1$, and $M_{1}>0$. Let $\omega_{0}: \mathbb{K} \times[0, \infty) \rightarrow[0, \infty)$ be continuous such that $(1.7)$ holds and $\left\|\omega_{0}\right\|_{\infty} \leq M_{1}$. We denote $\mathbb{U}_{\Omega, \mathbb{K}}\left(\Lambda, M_{0}, M_{1}, \omega_{0}\right)$ be the set consisting all vector fields $\mathbb{A}: \Omega \times \mathbb{K} \times \mathbb{R}^{n} \rightarrow \mathbb{R}^{n}$ such that (1.2)-(1.8) hold for some weight function $\mu \in A_{2}$ with $[\mu]_{A_{2}} \leq M_{0}$. Moreover, with a given $\mathbb{A} \in \mathbb{U}_{\Omega, \mathbb{K}}\left(\Lambda, M_{0}, M_{1}, \omega_{0}\right)$, the matrix $\tilde{\mathbb{A}}$ defined in (1.6) is called asymptotical matrix of $\mathbb{A}$.

We now recall what it means by weak solutions.

Definition 1.2. (i) For some $R>0$, let $B_{R}(y)$ be any ball of radius $R$ centered at $y \in \mathbb{R}^{n}$. Assume $\mathbb{A} \in \mathbb{U}_{B_{R}(y), \mathbb{K}}\left(\Lambda, M_{0}, M_{1}, \omega_{0}\right)$ with its asymptotical matrix $\widetilde{\mathbb{A}}$ and weight $\mu$. Let $\mathbf{F}: B_{R}(y) \rightarrow \mathbb{R}^{n}$ be a vector field such that $\mathbf{F} / \mu \in L^{2}\left(B_{R}(y), \mu\right)$. A function $u \in W^{1,2}\left(B_{R}(y), \mu\right)$ is said to be a weak solution of

$$
\operatorname{div}\left[\mathbb{A}(x, u, \nabla u]=\operatorname{div}[\mathbf{F}], \quad \text { in } \quad B_{R}(y),\right.
$$

if $u(x) \in \mathbb{K}$ for a.e. $x \in B_{R}(y)$, and

$$
\int_{B_{R}(y)}\langle\mathbb{A}(x, u, \nabla u), \nabla \varphi\rangle d x=\int_{B_{R}(y)}\langle\mathbf{F}, \nabla \varphi\rangle d x, \quad \forall \varphi \in C_{0}^{\infty}\left(B_{R}(y)\right) .
$$


(ii) Assume $\mathbb{A} \in \mathbb{U}_{\Omega, \mathbb{K}}\left(\Lambda, M_{0}, M_{1}, \omega_{0}\right)$ with its asymptotical matrix $\tilde{\mathbb{A}}$ and weight $\mu$. Let $g \in W^{1,2}(\Omega, \mu)$, $\mathbf{F}: \Omega \rightarrow \mathbb{R}^{n}$ be a vector field such that $\mathbf{F} / \mu \in L^{2}(\Omega, \mu)$. A function $u \in W^{1,2}(\Omega, \mu)$ is said to be a weak solution of (1.1) if $u-g \in W_{0}^{1,2}(\Omega, \mu), u(x) \in \mathbb{K}$ for a.e. $x \in \Omega$, and

$$
\int_{\Omega}\langle\mathbb{A}(x, u, \nabla u), \nabla \varphi\rangle d x=\int_{\Omega}\langle\mathbf{F}, \nabla \varphi\rangle d x, \quad \forall \varphi \in C_{0}^{\infty}(\Omega) .
$$

For any integrable function $f$ on a measurable $E \subset \mathbb{R}^{n}$, we also denote the average of $f$ on $E$ as

$$
\langle f\rangle_{E}=\frac{1}{|E|} \int_{E} f(y) d y, \quad \text { with } \quad|E|=\int_{E} d x .
$$

Moreover, with a measurable set $E \subset \mathbb{R}^{n}$ and a positive Borel measure $\mu$, we also denote

$$
\mu(E)=\int_{E} d \mu(x) .
$$

At this moment, we refer the readers to the definitions of $A_{p}$ classes of Muchkenhoupt weights and pairs of weights satisfying the $q$-Sawyer's condition in Definition 2.1, and Definition 2.5. Our first result is the interior estimates for the gradients of weak solutions of (1.1).

Theorem 1.3. Let $\Lambda>0, M_{0}, q \geq 1, M_{1}>0, M_{2} \geq 1$ and $p>2$. Then there is $\delta=\delta\left(q, \Lambda, M_{0}, M_{1}, M_{2}, p, n\right) \in$ $(0,1 / 8)$ and sufficiently small such that the following holds: Suppose that $\mathbb{K} \subset \mathbb{R}$ is an open interval and $\omega_{0}: \mathbb{K} \times[0, \infty) \rightarrow[0, \infty)$ is continuous satisfying (1.7) and $\left\|\omega_{0}\right\|_{\infty} \leq M_{1}$. For some $R>0$, let $\mathbb{A} \in \mathbb{U}_{B_{2 R}, \mathbb{K}}\left(\Lambda, M_{0}, M_{1}, \omega_{0}\right)$, with its asymptotical matrix $\tilde{\mathbb{A}}$, and weight $\mu \in A_{2}$. Suppose that $\tilde{\mathbb{A}}$ satisfies the following smallness condition on bounded mean oscillation with respect to the weight $\mu$ :

$$
\sup _{0<r<R_{0}} \sup _{x \in B_{R}} \frac{1}{\mu\left(B_{r}(x)\right)} \int_{B_{r}(x)}\left|\tilde{\mathbb{A}}(y)-\langle\tilde{\mathbb{A}}\rangle_{B_{r}(x)}\right|^{2} \mu^{-1}(y) d y \leq \delta, \quad \text { for some } \quad R_{0} \in(0, R] .
$$

Then, if $u \in W^{1,2}\left(B_{2 R}, \mu\right)$ is a weak solution of

$$
\operatorname{div}[\mathbb{A}(x, u, \nabla u)]=\operatorname{div}[\mathbf{F}], \quad \text { in } B_{2 R},
$$

and if $\omega \in A_{q}$ with $[\omega]_{A_{q}} \leq M_{2}$, and the pair $(\mu, \omega)$ satisfies the $\frac{p}{2}$-Sawyer's condition, then there is a constant $C=C\left(p, q, \Lambda, M_{0}, M_{1}, M_{2}, \omega_{0}, n\right)>0$ such that the estimate

$$
\begin{aligned}
& \left(\frac{1}{\omega\left(B_{R}\right)} \int_{B_{R}}|\nabla u|^{p} \omega(x) d x\right)^{1 / p} \\
& \leq C\left[\left(\frac{1}{\mu\left(B_{2 R}\right)} \int_{B_{2 R}}|\nabla u|^{2} \mu(x) d x\right)^{1 / 2}+\left(\frac{1}{\omega\left(B_{2 R}\right)} \int_{B_{2 R}}|\mathbf{F} / \mu|^{p} \omega(x) d x\right)^{1 / p}+1\right],
\end{aligned}
$$

holds, assuming that $|\mathbf{F} / \mu| \in L^{2}\left(B_{2 R}, \mu\right) \cap L^{p}\left(B_{2 R}, \omega\right)$.

We now have some discussion regarding (1.10). We emphasize that in $(1.10)$, the average $\langle\tilde{\mathbb{A}}\rangle_{B_{r}(x)}$ of $\tilde{\mathbb{A}}$ in the ball $B_{r}(x)$ is taken with respect the Lebesgue measure. The metric defined as in (1.10) is introduced in [19, 20, 32, 33] and called mean oscillation with respect to the weight $\mu$. A space consisting of all locally integrable functions satisfying the bounded mean oscillation with respect to some fixed given weight is also studied in [19, 20, 32, 33]. As noted in [19, 20, 32, 33], this space is different from the usual weighted BMO space, and is also different from the well-known John-Nirenberg BMO space. We also note that the smallness condition (1.10) is natural as it reduces to the regular smallness condition in BMO space that already used in known work [1, 3, 5, 6, 7, 8, 9, 12, 13, 23, 24, 25, 26, 27, 28, 29, 21, 44]. Moreover, as demonstrated by a counterexample in [8], the smallness condition (1.10) is necessary. In particular, as it is shown in [8], the estimate (1.11) is even not valid when the coefficients $\tilde{\mathbb{A}}$ is uniformly continuous, but degenerate. In this light and compared to [14], Theorem 1.3, once reduced to the linear case, gives the right conditions on the coefficients so that the $W^{1, p}$-regularity estimates hold.

To derive the $L^{p}$-estimates for solutions up to the boundary, we need some regularity condition on $\partial \Omega$. The following definition is important in the paper. 
Definition 1.4. We say that $\Omega$ is a $\left(\delta, R_{0}\right)$-Reifenberg flat domain if, for every $x \in \partial \Omega$ and every $r \in\left(0, R_{0}\right)$, there exists a coordinate system $\left\{\vec{y}_{1}, \vec{y}_{2}, \cdots, \vec{y}_{n}\right\}$ which may depend on $x$ and $r$, such that in this coordinate system $x=0$ and

$$
B_{r}(0) \cap\left\{y_{n}>\delta r\right\} \subset B_{r}(0) \cap \Omega \subset B_{r}(0) \cap\left\{y_{n}>-\delta r\right\} .
$$

In the above and hereafter $B_{\rho}(x)$ denotes a ball of radius $\rho$ centered at $x, B_{\rho}^{+}(x)$ is the upper-half ball, and $\Omega_{\rho}(x)=B_{\rho}(x) \cap \Omega$, the portion of the ball in $\Omega$. We also need the following additional definition in order to derive the local estimate of $\nabla u$ on the boundary.

Definition 1.5. Let $\Omega \subset \mathbb{R}^{n}$ be a bounded domain. Let $R>0$ and $y_{0} \in \bar{\Omega}$. The domain $\Omega_{R}\left(y_{0}\right)=\Omega \cap B_{R}\left(y_{0}\right)$ is said to be of type $(A, r)$, if there is $A>0$ and $r \in(0, R / 2)$ such that for every $y \in \Omega_{R}\left(y_{0}\right)$, and $\rho \in(0, r)$ if $B_{\rho}(y) \cap \partial B_{R}\left(y_{0}\right) \cap \bar{\Omega} \neq \emptyset$, and $B_{\rho}(y) \cap \partial \Omega \cap \bar{B}_{R}\left(y_{0}\right) \neq \emptyset$ then

$$
\left|\Omega_{R}\left(y_{0}\right) \cap B_{\rho}(y)\right| \geq A\left|B_{\rho}(y)\right| .
$$

We remark that if $\bar{\Omega} \subset B_{R}\left(y_{0}\right)$, then $\Omega_{R}\left(y_{0}\right)=\Omega$ and it is a $(1, r)$ domain with $r \in\left(0\right.$, $\left.\operatorname{dist}\left(\partial \Omega, B_{R}\left(y_{0}\right)\right)\right)$. Moreover, if $\Omega=B_{2}^{+}(0)$, then $\Omega_{1}(0)$ is of type $(A, r)$ with some $A=A(n)>0$ and $r \in(0,1)$. The following theorem is the main result of the paper.

Theorem 1.6. Let $\Lambda>0, A>0, M_{0}, q \geq 1, M_{1}>0, M_{2} \geq 1$, and $p>2$. Then there is a sufficiently small number $\delta \in(0,1 / 8)$ depending on $p, q, \Lambda, A, M_{0}, M_{1}, M_{2}$, and $n$ such that the following holds: Suppose that $\Omega$ is a $\left(\delta, R_{0}\right)$-Reifenberg flat domain for some $R_{0}>0, \mathbb{K} \subset \mathbb{R}$ is an open interval, and $\omega_{0}: \mathbb{K} \times[0, \infty) \rightarrow$ $[0, \infty)$ is continuous satisfying (1.7) and $\left\|\omega_{0}\right\|_{\infty} \leq M_{1}$. Suppose also that $\mathbb{A} \in \mathbb{U}_{\Omega, \mathbb{K}}\left(\Lambda, M_{0}, M_{1}, \omega_{0}\right)$, with its asymptotical matrix $\tilde{\mathbb{A}}$, and weight $\mu \in A_{2}$ satisfying the following smallness condition on bounded mean oscillation with respect to the weight $\mu$ :

$$
\sup _{0<r<R_{0}} \sup _{x \in \Omega} \frac{1}{\mu\left(B_{r}(x)\right)} \int_{B_{r}(x) \cap \Omega}\left|\tilde{\mathbb{A}}(y)-\langle\tilde{\mathbb{A}}\rangle_{B_{r}(x) \cap \Omega}\right|^{2} \mu^{-1}(y) d y \leq \delta .
$$

Then, for every $R>0, y_{0} \in \bar{\Omega}$ such that $\partial \Omega \cap B_{R}\left(y_{0}\right) \neq \emptyset$, and $\Omega_{R}\left(y_{0}\right)$ is of type $\left(A, r_{0}\right)$ with some small number $r_{0}>0$, and for $\omega \in A_{q}$ with $[\omega]_{A_{q}} \leq M_{2}$, and the pair $(\mu, \omega)$ satisfies the $\frac{p}{2}$-Sawyer's condition, there is some positive constant $C=C\left(p, q, \Lambda, A, M_{0}, M_{1}, M_{2}, \Omega, R / r_{0}, \omega_{0}, n\right)$ such that if $u \in W^{1,2}(\Omega, \mu)$ is a weak solution of (1.1), the estimate

$$
\begin{aligned}
\int_{\Omega_{R}\left(y_{0}\right)}|\nabla u|^{p} \omega(x) d x \leq C & {\left[\int_{\Omega_{2 R}\left(y_{0}\right)}|\nabla g|^{p} \omega(x) d x+\int_{\Omega_{2 R}\left(y_{0}\right)}|\mathbf{F} / \mu|^{p} \omega(x) d x\right.} \\
& \left.+\omega\left(\Omega_{R}\left(y_{0}\right)\right)\left\{\left(\frac{1}{\mu\left(B_{2 R}\left(y_{0}\right)\right)} \int_{\Omega_{2 R}\left(y_{0}\right)}|\nabla u|^{2} \mu(x) d x\right)^{p / 2}+1\right\}\right]
\end{aligned}
$$

holds if $|\nabla g| \in L^{2}(\Omega, \mu) \cap L^{p}\left(\Omega_{2 R}\left(y_{0}\right), \omega\right)$, and $|\mathbf{F} / \mu| \in L^{2}(\Omega, \mu) \cap L^{p}\left(\Omega_{2 R}\left(y_{0}\right), \omega\right)$.

A few comments on Theorem 1.3 and Theorem 1.6 are now in ordered. Firstly, as we already discussed, the smallness condition (1.12) is optimal, as there are counterexamples given in [8] for the degenerate case, and [29] for the uniformly elliptic case. Secondly, we emphasize that the novelty in Theorem 1.3 and Theorem 1.6 is that the coefficients $\mathbb{A}$ are non-uniformly elliptic and they could depend on $u$. Moreover, unlike the previous work [2, 17, 36, 37], Theorem 1.3 and Theorem 1.6 do not assume the boundedness nor continuity of the weak solutions $u$. Therefore, even in case that $\mathbb{A}$ is uniformly elliptic, Theorem 1.3 , and Theorem 1.6 are already new. Lastly, we would like to point out that the type of local $W^{1, p}$ - estimates near the boundary as Theorem 1.6 are necessary in many applications in which only local information of the data near the considered point on the boundary is known. Also, observe that Reifenberg flat domains are already used in previous work [1, 3, 5, 6, 27, 28]. However, the local estimates near the boundary as stated in Theorem 1.6 do not seem to appear, nor to be produced directly from the global $W^{1, p}$-estimate results in these mentioned papers, even for smooth domains. Theorem 1.6 bridges both versions of local and global 


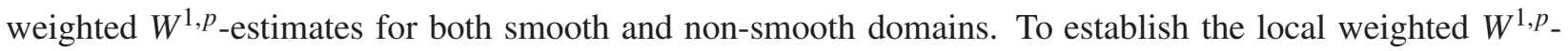
estimates near the boundary $y_{0} \in \partial \Omega$ for non-smooth Reifenberg flat domains, we impose the $\left(A, r_{0}\right)$-type condition on $\Omega_{R}\left(y_{0}\right)$. Several technical analysis issues are revised and improved to prove Theorem 1.6 ,

There are two major difficulties in proving Theorems 1.3 1.6. The first one is due to the scaling properties the equations. Observe that the class of equations (1.1) is not invariant under the usual scalings $u \rightarrow u / \lambda$ and the dilations $u(x) \rightarrow u(r x) / r$ with positive numbers $\lambda, r$. This is very serious since the theory of Sobolev regularity estimates for weak solutions relies heavily on these scalings and dilations, see for instance [1, 3, 5, 6, 9, 12, 13, 23, 24, 25, 26, 27, 28, 29, 21, 44]. We overcome this by adapting the perturbation technique with double-scaling parameter method introduced in [17], see also [36, 37] for the implementation of the method. In this perspective, the following observation regarding the scaling property of (1.1) is essential in the paper.

Remark 1.7. Let $\mathbb{A} \in \mathbb{U}_{\Omega, \mathbb{K}}\left(\Lambda, M_{0}, M_{1}, \omega_{0}\right)$ with its corresponding asymptotical matrix $\tilde{\mathbb{A}}$, weight $\mu \in A_{2}$. Let $\lambda>0$ and define $\mathbb{K}_{\lambda}=\mathbb{K} / \lambda$ and

$$
\mathbb{A}_{\lambda}(x, z, \eta)=\mathbb{A}(x, \lambda z, \lambda \eta) / \lambda, \quad(x, z, \eta) \in \Omega \times \mathbb{K}_{\lambda} \times \mathbb{R}^{n} .
$$

Then, it is simple to check that

$$
\left|\mathbb{A}_{\lambda}(x, z, \eta)-\tilde{\mathbb{A}}(x) \eta\right| \leq \frac{1}{\lambda} \omega_{0}(\lambda z, \lambda \eta)(1+|\lambda \eta|) \mu(x), \quad \forall(x, z, \eta) \in \Omega \times \mathbb{K}_{\lambda} \times \mathbb{R}^{n} .
$$

The other major difficulty is due to the fact that we are working with two different weights, i.e. $\mu$ and $\omega$. On one hand, all natural intermediate step estimates such as energy estimates, weighted maximal function operators $\mathcal{M}_{\mu}\left(|\nabla u|^{2}\right)$, and approximation estimates are performed in weighted spaces with weight $\mu$. On the other hand, to obtain the estimates of $|\nabla u|$ in $L^{p}(\Omega, \omega)$, it requires to control the level sets, and density estimates of level sets of $\mathcal{M}_{\mu}\left(|\nabla u|^{2}\right)$ in measure $\omega$. Therefore, it requires to interchange the two measures. Lemma 2.3 below serves as the key ingredient for some kind of weak type $(1,1)$ estimates of two different weights. The lemma is indeed a simple consequence of the Coifman-Feferrman's result [10] on the reverse Hölder's inequality, and the doubling properties of the Muckenhoupt weights. Theorem 1.3, and Theorem 1.6 therefore can be viewed as a two weighted nonlinear Calderón-Zygmund type estimates.

We conclude the section by highlighting the layout of the paper. Some analysis preliminary tools in measure theories and weighted norm inequalities are reviewed in the next section, Section 2 Section 3 consists interior intermediate step estimates and the proof of Theorem 1.3 . Section 4 treats the estimates near the boundary points and then proves Theorem 1.6 .

\section{Preliminaries on Weights and Weighted NORm INequalities}

This section recalls several real analysis results, definitions needed in the paper. We first recall the definition of $A_{p}$ - Muckenhoupt class of weights introduced in [34].

Definition 2.1. Let $1 \leq p<\infty$, a non-negative, locally integrable function $\omega: \mathbb{R}^{n} \rightarrow[0, \infty)$ is said to be in the class $A_{p}$ of Muckenhoupt weights if

$$
\begin{aligned}
& {[\omega]_{A_{p}}:=\sup _{\text {balls } B \subset \mathbb{R}^{n}}\left(f_{B} \omega(x) d x\right)\left(f_{B} \omega(x)^{\frac{1}{1-p}} d x\right)^{p-1}<\infty, \text { if } p>1,} \\
& {[\omega]_{A_{1}}:=\sup _{\text {balls } B \subset \mathbb{R}^{n}}\left(f_{B} \omega(x) d x\right)\left\|\omega^{-1}\right\|_{L^{\infty}(B)}<\infty \text { if } p=1 .}
\end{aligned}
$$

It turns out that the class of $A_{p}$-Muckenhout weights satisfies the reverse Hölder's inequality and the doubling properties. In particular, a measure of any $A_{p}$-weight is comparable with the Lebesgue measure. This is in fact a well-known result due to R. Coifman and C. Fefferman [10], and it is an important ingredient in the paper.

Lemma 2.2 ([10]). For $1<p<\infty$, the following statements hold true 
(i) If $\mu \in A_{p}$, then for every ball $B \subset \mathbb{R}^{n}$ and every measurable set $E \subset B$,

$$
\mu(B) \leq[\mu]_{A_{p}}\left(\frac{|B|}{|E|}\right)^{p} \mu(E) .
$$

(ii) If $\mu \in A_{p}$ with $[\mu]_{A_{p}} \leq M$ for some given $M \geq 1$, then there is $C=C(M, n)$ and $\beta=\beta(M, n)>0$ such that

$$
\mu(E) \leq C\left(\frac{|E|}{|B|}\right)^{\beta} \mu(B)
$$

for every ball $B \subset \mathbb{R}^{n}$ and every measurable set $E \subset B$.

From Lemma2.2, we can infer that any two Muckenhoupt weights are comparable. The following lemma is a consequence of Lemma2.2 and it is used frequently in this paper.

Lemma 2.3. Let $1<q, s<\infty$ and $M \geq 1, M^{\prime} \geq 1$ Assume that $\mu \in A_{s}$ and $\omega \in A_{q}$ such that

$$
[\mu]_{A_{s}} \leq M, \quad[\omega]_{A_{q}} \leq M^{\prime} .
$$

Then, there exists $\beta=\beta\left(M^{\prime}, n\right)>0$ such that for every ball $B$ and every measurable set $E \subset B$,

$$
\omega(E) \leq C\left(M, M^{\prime}, s, n\right)\left(\frac{\mu(E)}{\mu(B)}\right)^{\beta / s} \omega(B)
$$

Proof. By Lemma2.2 -(ii), there is $\beta=\beta\left(M^{\prime}, n\right)>0$ such that

$$
\frac{\omega(E)}{\omega(B)} \leq C\left(M^{\prime}, n\right)\left(\frac{|E|}{|B|}\right)^{\beta} .
$$

On the other hand, by Lemma2.2-(i), we see that

$$
\left(\frac{|E|}{|B|}\right)^{s} \leq M \frac{\mu(E)}{\mu(B)}
$$

Combining the two estimates, we obtain

$$
\frac{\omega(E)}{\omega(B)} \leq C\left(M, M^{\prime}, s, n\right)\left(\frac{\mu(E)}{\mu(B)}\right)^{\beta / s},
$$

as desired.

Next, we state a standard result in measure theory.

Lemma 2.4. Assume that $g \geq 0$ is a measurable function in a bounded subset $U \subset \mathbb{R}^{n}$. Let $\theta>0$ and $N>1$ be given constants. If $\mu$ is a weight function in $\mathbb{R}^{n}$, then for any $1 \leq p<\infty$

$$
g \in L^{p}(U, \mu) \Leftrightarrow S:=\sum_{j \geq 1} N^{p j} \mu\left(\left\{x \in U: g(x)>\theta N^{j}\right\}\right)<\infty .
$$

Moreover, there exists a constant $C>0$ depending only on $\theta, N$ and $p$. such that

$$
C^{-1} S \leq\|g\|_{L^{p}(U, \mu)}^{p} \leq C(\mu(U)+S) .
$$

Now, we discuss about the weighted Hardy-Littlewood maximal operator and its boundedness in weighted spaces. For a given locally integrable function $f$ and a weight $\mu$, we define the weighted Hardy-Littlewood maximal function as

$$
\mathcal{M}_{\mu} f(x)=\sup _{\rho>0} f_{B_{\rho}(x)}|f(y)| d(\mu(y)) .
$$

For functions $f$ that are defined on a bounded domain, we define

$$
\mathcal{M}_{\mu, \Omega} f(x)=\mathcal{M}_{\mu}\left(f \chi_{\Omega}\right)(x) .
$$

We introduce the definition $p$-pair of weights satisfying Sawyer's condition. 
Definition 2.5. Let $\mu, \omega$ be any two positive, locally finite Borel measures on $\mathbb{R}^{n}$ and let $1<p<\infty$. The pair of measures $(\mu, \omega)$ is said to satisfy the $p$-Sawyer's condition if there is a constant $C>0$ such that

$$
\int_{B}\left(\mathcal{M}_{\mu}\left(\chi_{B} \frac{d \sigma}{d \mu}\right)\right)^{p} d \omega \leq C \sigma(B), \quad \forall \text { ball } B \subset \mathbb{R}^{n},
$$

where $\chi_{B}$ is the characteristic function of the ball $B$,

$$
d \sigma=(d \mu / d \omega)^{p^{\prime}} d \omega, \quad \text { and } \quad \frac{1}{p}+\frac{1}{p^{\prime}}=1
$$

The following result is due to E. R. Sawyer in [39]. Other proofs of this result can be found in [11, 43].

Theorem 2.6. Let $\mu, \omega$ be any two positive, locally finite Borel measures on $\mathbb{R}^{n}$ and let $1<q<\infty$. Then,

$$
\left\|\mathcal{M}_{\mu}\right\|_{L^{q}\left(\mathbb{R}^{n}, \omega\right) \rightarrow L^{q}\left(\mathbb{R}^{n}, \omega\right)} \leq C,
$$

if and only if the pair $(\mu, \omega)$ satisfies the q-Sawyer's condition.

We note that if $d \mu(x)=d x$, i.e. $\mu=1$, it follows from [18, 34], see also [40, Theorem 1, p. 201], that the pair $(1, \omega)$ satisfies the $q$-Sawyer's condition if and only if the weight function $\omega \in A_{q}$. Moreover, if $\mu$ is a doubling measure, the pair $(\mu, \mu)$ always satisfies the $q$-Sawyer's condition.

We next state the following modified version of the Vitali's covering lemma, which is needed in the paper. The proof of this lemma can be found in the Appendix A at the end of the paper.

Lemma 2.7. Suppose that $\Omega$ is a $\left(\delta, R_{0}\right)$-Reifenberg flat domain with some small numbers $\delta \in(0,1 / 8)$ and $R_{0}>0$. Let $\omega \in A_{q}$ be a weight for some $q \in(1, \infty)$ with $[\omega]_{A_{p}} \leq M, R>0$, and $y_{0} \in \bar{\Omega}$. Assume that $\Omega_{R}\left(y_{0}\right)$ is of type $\left(A, r_{0}\right)$ with some $r_{0} \in\left(0, \min \left\{R_{0}, R\right\} / 2\right), C, D$ are measurable sets satsifying $C \subset D \subset \Omega_{R}\left(y_{0}\right)$, and there exists $0<\epsilon<1$ such that

(i) $\omega(C)<\epsilon \omega\left(B_{r_{0}}(y)\right)$ for almost every $y \in \Omega_{R}\left(y_{0}\right)$, and

(ii) for all $x \in \Omega_{R}\left(y_{0}\right)$ and $\rho \in\left(0, r_{0}\right)$, if $\omega\left(C \cap B_{\rho}(x)\right) \geq \epsilon \omega\left(B_{\rho}(x)\right)$, then $B_{\rho}(x) \cap \Omega_{R}\left(y_{0}\right) \subset D$.

Then the estimate

$$
\omega(C) \leq \epsilon^{\prime} \omega(D), \quad \text { for } \quad \epsilon^{\prime}=\epsilon 5^{n q} \max \left\{A^{-1}, 4^{n}\right\}^{q} M^{2} .
$$

A simple version of Lemma 2.7 with $\Omega=B_{R}$ is also used in the paper for the interior estimates, we therefore state it here for later reference.

Lemma 2.8. Suppose $\omega \in A_{q}$ with some $1<q<\infty$, and suppose that $R>0, C, D$ are measurable sets satisfying $C \subset D \subset B_{R}$. Assume also that there are $r_{0} \in(0, R / 2)$, and $0<\epsilon<1$ such that

(i) $\omega(C)<\epsilon \omega\left(B_{r_{0}}(y)\right)$ for almost every $y \in B_{R}$, and

(ii) for all $x \in B_{R}\left(y_{0}\right)$ and $\rho \in\left(0, r_{0}\right)$, if $\omega\left(C \cap B_{\rho}(x)\right) \geq \epsilon \omega\left(B_{\rho}(x)\right)$, then $B_{\rho}(x) \cap B_{R} \subset D$.

Then

$$
\omega(C) \leq \epsilon^{\prime} \omega(D), \quad \text { for } \quad \epsilon^{\prime}=\epsilon 20^{n q}[\omega]_{A_{q}}^{2} .
$$

Finally, we state a remark which follows directly from Definition 1.4 about Reifenberg flat domains, see [28, Remark 3.2] for details.

Remark 2.9. If $\Omega$ is a $\left(\delta, R_{0}\right)$ flat domain with $\delta \in(0,1 / 2)$, then for any point $x$ on the boundary and $\rho \in\left(0, R_{0} / 2\right)$, there exists a coordinate system $\vec{z}_{1}, \vec{z}_{2}, \cdots, \vec{z}_{n}$ with the origin at some point in the interior of $\Omega$ such that in this coordinate system $x=-\delta \rho \vec{z}_{n}$ and

$$
B_{\rho}^{+}(0) \subset \Omega_{\rho} \subset B_{\rho}(0) \cap\left\{\left(z_{1}, \cdots, z_{n-1}, z_{n}\right): z_{n}>-4 \delta \rho\right\} .
$$




\section{INTERIOR $W^{1, p}$ - REGULARITY THEORY}

3.1. Interior approximation estimates. In this section, let $r>0$ and an open interval $\mathbb{K} \subset \mathbb{R}$. For $\mathbb{A} \in$ $\mathbb{U}_{B_{r}, \mathbb{K}}\left(\Lambda, M_{0}, M_{1}, \omega_{0}\right)$, let $\mathbb{A}_{\lambda}$ be defined as in Remark 1.7 with some $\lambda>0$. We focus on the equation

$$
\operatorname{div}\left[\mathbb{A}_{\lambda}(x, u, \nabla u)\right]=\operatorname{div}[\mathbf{F}], \quad \text { in } \quad B_{r},
$$

We need to state what we mean by weak solution of (3.1).

Definition 3.1. A function $u \in W^{1,2}\left(B_{r}, \mu\right)$ is said to be a weak solution of (3.1) if $u(x) \in \mathbb{K}_{\lambda}:=\mathbb{K} / \lambda$ for all most every $x \in B_{r}$ and

$$
\int_{B_{r}}\left\langle\mathbb{A}_{\lambda}(x, u, \nabla u), \nabla \varphi\right\rangle d x=\int_{B_{r}}\langle\mathbf{F}, \nabla \varphi\rangle d x
$$

holds for all $\varphi \in C_{0}^{\infty}\left(B_{r}\right)$.

Let $\tilde{\mathbb{A}}$ be the asymptotical matrix of $\mathbb{A}$ with the asymptotical weight function $\mu \in A_{2}$, and we recall that

$$
\Lambda^{-1}|\eta|^{2} \mu(x) \leq\langle\tilde{\mathbb{A}}(x) \eta, \eta\rangle \leq \Lambda|\eta|^{2} \mu(x), \quad \text { for a.e. } x \in B_{r}, \quad \forall \eta \in \mathbb{R}^{n} .
$$

and

$$
[\mu]_{A_{2}} \leq M_{0}
$$

The following Proposition is the main result of the section.

Proposition 3.2. Let $\Lambda>0, M_{0} \geq 1, M_{1}>0$ and a continuous function $\omega_{0}$ satisfying (1.7), $\left\|\omega_{0}\right\|_{\infty} \leq$ $M_{1}$. Then, for every small number $\epsilon>0$, there exist $\delta=\delta\left(\epsilon, \Lambda, M_{0}, n\right)>0$ sufficiently small and $\lambda=$ $\lambda\left(\epsilon, \Lambda, M_{0}, M_{1}, \omega_{0}, n\right) \geq 1$ such that the following holds: Assume that $\mathbb{A} \in \mathbb{U}_{B_{r}(y), \mathbb{K}}\left(\Lambda, M_{0}, M_{1}, \omega_{0}\right)$, for some $r>0, y \in \mathbb{R}^{n}$, some open interval $\mathbb{K} \subset \mathbb{R}$, and with its asymptotical matrix $\tilde{\mathbb{A}}$, and weight $\mu \in A_{2}$. Assume also that

$$
\frac{1}{\mu\left(B_{r}(y)\right)} \int_{B_{r}(y)}\left|\tilde{\mathbb{A}}(x)-\langle\tilde{\mathbb{A}}\rangle_{B_{r}(y)}\right|^{2} \mu^{-1}(x) d x \leq \delta . \quad \text { and } \quad \frac{1}{\mu\left(B_{7 r / 8}(y)\right)} \int_{B_{7 r / 8}(y)}\left|\frac{\mathbf{F}}{\mu}\right|^{2} \mu(x) d x \leq \delta .
$$

Then, for every $\lambda \geq \lambda_{0}$, if $u \in W^{1,2}\left(B_{r}(y), \mu\right)$ is a weak solution of

$$
\operatorname{div}\left[\mathbb{A}_{\lambda}(x, u, \nabla u)\right]=\operatorname{div}[\mathbf{F}], \quad \text { in } B_{r}(y)
$$

satisfying

$$
\frac{1}{\mu\left(B_{7 r / 8}(y)\right)} \int_{B_{7 r / 8}(y)}|\nabla u|^{2} \mu(x) d x \leq 1,
$$

then there is a Lipschitz function $v$ defined on $B_{3 r / 4}(y)$, and constant $C=C\left(\Lambda, M_{0}, n\right)$ such that

$$
\frac{1}{\mu\left(B_{r / 2}(y)\right)} \int_{B_{r / 2}(y)}|\nabla u-\nabla v|^{2} \mu(x) d x \leq \epsilon, \quad \text { and } \quad\|\nabla v\|_{L^{\infty}\left(B_{r / 2}(y)\right)} \leq C .
$$

More precisely, $v \in W_{\mathrm{loc}}^{1,2}\left(B_{3 r / 4}(y)\right)$ is a weak solution of the equation

$$
\operatorname{div}\left[\mathbb{A}_{0} \nabla v\right]=0, \quad \text { in } \quad B_{\frac{3 r}{4}}(y)
$$

for some constant symmetric matrix $\mathbb{A}_{0}$ satisfying $\left|\langle\tilde{\mathbb{A}}\rangle_{B_{r}(y)}-\mathbb{A}_{0}\right| \leq \epsilon \frac{\mu\left(B_{r}(y)\right)}{\left|B_{r}(y)\right|}$.

The rest of the section is to prove Proposition 3.2. By using translation $x \rightarrow x-y$, we can assume $y=0$. We split the procedure for the proof into two steps of approximations. 
3.1.1. First approximation estimates. Our first step is to approximate the solution $u$ of (3.1) by the solution $w$ of the following equation

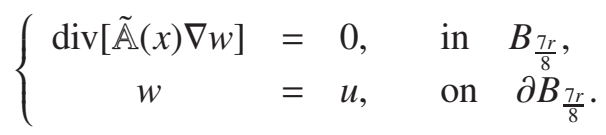

We note that for a given $u \in W^{1,2}\left(B_{7 r / 8}, \mu\right)$, it follows from [15, Theorem 2.2] that there exists a unique weak solution $w \in W^{1,2}\left(B_{7 r / 8}, \mu\right)$ of (3.4). Our result of this section is the following weighted energy estimate for the weak solutions $u, w$.

Lemma 3.3. Assume that $u \in W^{1,2}\left(B_{r}\right)$ is a weak solution of (3.1) with some $\lambda>0$ and let $w \in W^{1,2}\left(B_{\frac{7 r}{8}}, \mu\right)$ be the weak solution of (3.4). Then, there is a constant $C(\Lambda)>0$ such that

$$
f_{B_{\frac{7 r}{8}}}|\nabla w|^{2} d \mu(x) \leq C(\Lambda) f_{\frac{B_{\frac{7 r}{8}}}{}}|\nabla u|^{2} d \mu(x) .
$$

Moreover, for every $\delta>0$, there is $K_{\delta}>0$ depending only on $\delta$ and $\omega_{0}$ such that

$$
f_{B_{\frac{7 r}{8}}}|\nabla(u-w)|^{2} d \mu(x) \leq C(\Lambda)\left[\delta^{2} f_{B_{\frac{7 r}{8}}}|\nabla u|^{2} d \mu(x)+f_{B_{\frac{7 r}{8}}}\left|\frac{\mathbf{F}}{\mu}\right|^{2} d \mu(x)+\frac{\left(M_{1} K_{\delta}+\delta\right)^{2}}{\lambda^{2}}\right] .
$$

Proof. We write $\theta=\frac{7 r}{8}$. Since $u-w \in W_{0}^{1,2}\left(B_{\theta}, \mu\right)$, we can use it as a test function for (3.4) to obtain

$$
\int_{B_{\theta}}\langle\tilde{\mathbb{A}}(x) \nabla w, \nabla w\rangle d x=\int_{B_{\theta}}\langle\tilde{\mathbb{A}}(x) \nabla w, \nabla u\rangle d x .
$$

This and (3.2) imply

$$
\begin{aligned}
\Lambda^{-1} \int_{B_{\theta}}|\nabla w|^{2} \mu(x) d x & \leq \Lambda \int_{\theta}|\nabla u \| \nabla w| \mu(x) d x \\
& \leq \frac{\Lambda^{-1}}{2} \int_{B_{\theta}}|\nabla w|^{2} \mu(x) d x+C(\Lambda) \int_{B_{\theta}}|\nabla u|^{2} \mu(x) d x .
\end{aligned}
$$

Therefore,

$$
\int_{B_{\theta}}|\nabla w|^{2} \mu(x) d x \leq C(\Lambda) \int_{B_{\theta}}|\nabla u|^{2} \mu(x) d x .
$$

This estimate proves (3.7). Also, from (3.7) it follows that

$$
\begin{aligned}
& \int_{B_{\theta}}\langle\tilde{\mathbb{A}}(x) \nabla(u-w), \nabla(u-w)\rangle d x \\
& =\int_{B_{\theta}}\langle\tilde{\mathbb{A}}(x) \nabla u, \nabla u\rangle d x-2 \int_{B_{\theta}}\langle\tilde{\mathbb{A}}(x) \nabla u, \nabla w\rangle d x+\int_{B_{\theta}}\langle\tilde{\mathbb{A}}(x) \nabla w, \nabla w\rangle d x \\
& =\int_{B_{\theta}}\langle\tilde{\mathbb{A}}(x) \nabla u, \nabla u\rangle d x-\int_{B_{\theta}}\langle\tilde{\mathbb{A}}(x) \nabla u, \nabla w\rangle d x=\int_{B_{\theta}}\langle\tilde{\mathbb{A}}(x) \nabla u, \nabla u-\nabla w\rangle d x .
\end{aligned}
$$

On the other hand, use $u-w$ as a test function for (3.1), we obtain

$$
\int_{B_{\theta}}\left\langle\mathbb{A}_{\lambda}(x, u, \nabla u), \nabla(u-w)\right\rangle d x=\int_{B_{\theta}}\langle\mathbf{F}, \nabla(u-w)\rangle d x .
$$

Combining the last two equalities, we obtain

$$
\begin{aligned}
& \int_{B_{\theta}}\langle\tilde{\mathbb{A}}(x) \nabla(u-w), \nabla(u-w)\rangle d x \\
& =\int_{B_{\theta}}\left\langle\tilde{\mathbb{A}}(x) \nabla u-\mathbb{A}_{\lambda}(x, u, \nabla u), \nabla u-\nabla w\right\rangle d x-\int_{B_{\theta}}\langle\mathbf{F}, \nabla(u-w)\rangle d x .
\end{aligned}
$$


Therefore, it follows from (1.6)-(1.8), and (1.13) that

$$
\begin{aligned}
& \Lambda^{-1} \int_{B_{\theta}}|\nabla(u-w)|^{2} \mu(x) d x \\
& \leq \frac{1}{\lambda} \int_{B_{\theta}} \omega_{0}(\lambda u, \lambda|\nabla u|)(1+\lambda|\nabla u|)|\nabla u-\nabla w| \mu(x) d x+\int_{B_{\theta}}|\mathbf{F}||\nabla u-\nabla w| d x .
\end{aligned}
$$

It follows from (1.7) that for $\delta>0$, we can find a large number $K_{\delta}>0$ depending only on $\omega_{0}$ and $\delta$ such that $\omega_{0}(z, s) \leq \delta$ for all $s \geq K_{\delta}$ and for all $z \in \overline{\mathbb{K}}$. This and $\left\|\omega_{0}\right\|_{\infty} \leq M_{1}$ in turn imply that

$$
\omega_{0}(z, s)(1+s) \leq \delta(1+s)+M_{1} K_{\delta}, \quad \forall s \geq 0, \quad \forall z \in \mathbb{K},
$$

Hence,

$$
\begin{aligned}
& \Lambda^{-1} \int_{B_{\theta}}|\nabla(u-w)|^{2} \mu(x) d x \\
& \leq \int_{B_{\theta}}\left[\delta|\nabla u|+\lambda^{-1}\left(M_{1} K_{\delta}+\delta\right)\right]|\nabla u-\nabla w| \mu(x) d x+\int_{B_{\theta}}|\mathbf{F}||\nabla u-\nabla w| d x .
\end{aligned}
$$

Then, by using Hölder's inequality and Young's inequality, we get

$$
\begin{aligned}
& \Lambda^{-1} \int_{B_{\theta}}|\nabla(u-w)|^{2} \mu(x) d x \\
& \leq \epsilon \int_{B_{\theta}}|\nabla(u-w)|^{2} \mu(x)+C(\epsilon)\left[\delta^{2} \int_{B_{\theta}}|\nabla u|^{2} \mu(x) d x+\lambda^{-2}\left(M_{1} K_{\delta}+\delta\right)^{2} \mu\left(B_{\theta}\right)+\int_{B_{\theta}}\left|\frac{\mathbf{F}}{\mu}\right|^{2} \mu(x) d x\right] .
\end{aligned}
$$

Choosing $\epsilon<\Lambda^{-1} / 2$, we then obtain

$$
\int_{B_{\theta}}|\nabla(u-w)|^{2} \mu(x) d x \leq C(\Lambda)\left[\delta^{2} f_{B_{\theta}}|\nabla u|^{2} d \mu(x)+\lambda^{-2}\left(M_{1} K_{\delta}+\delta\right)^{2}+f_{B_{\theta}}\left|\frac{\mathbf{F}}{\mu}\right|^{2} d \mu(x)\right] \mu\left(B_{\theta}\right)
$$

This implies (3.6). The proof is now complete.

3.1.2. Second approximation estimates. Our second approximation is the following lemma.

Lemma 3.4. Let $\Lambda>0, M_{0}>1$. For every $\epsilon>0$ sufficiently small, there exists sufficiently small number $\delta^{\prime}=\delta^{\prime}\left(\epsilon, \Lambda, M_{0}, n\right)>0$ such that the following statement holds true: For some $r>0$, assume that (3.2) and (3.3) hold and

$$
\frac{1}{\mu\left(B_{r}\right)} \int_{B_{r}}\left|\tilde{\mathbb{A}}-\langle\tilde{\mathbb{A}}\rangle_{B_{r}}\right|^{2} \mu^{-1} d x \leq \delta^{\prime},
$$

then, for every weak solution $w \in W^{1,2}\left(B_{7 r / 8}, \mu\right)$ of

$$
\operatorname{div}[\tilde{\AA} \nabla w]=0, \quad \text { in } \quad B_{7 r / 8}
$$

satisfying $f_{B_{7 r / 8}}|\nabla w|^{2} d \mu \leq C_{0}(\Lambda)$, there exists a constant matrix $\mathbb{A}_{0}$ and a weak solution $v \in W_{\mathrm{loc}}^{1,2}\left(B_{3 r / 4}\right)$ of

$$
\operatorname{div}\left[\mathbb{A}_{0} \nabla v\right]=0, \quad \text { in } \quad B_{3 r / 4}
$$

such that

$$
\left|\langle\mathbb{A}\rangle_{B_{r}}-\mathbb{A}_{0}\right| \leq \frac{\epsilon \mu\left(B_{r}\right)}{\left|B_{r}\right|}, \quad \text { and } \quad f_{B_{r / 2}}|\nabla w-\nabla v|^{2} d \mu \leq \epsilon
$$

Moreover, there is $C=C\left(\Lambda, M_{0}, n\right)$ such that

$$
\|\nabla v\|_{L^{\infty}\left(B_{r / 2}\right)} \leq C .
$$

Proof. The proof is the same as that of [8, Proposition 4.4] with suitable scaling, we skip it. 
3.1.3. Proof of Proposition 3.2 We only need to glue Lemma 3.3 and Lemma 3.4 together. With given $\Lambda>0, M_{0} \geq 1$ and $\epsilon>0$, let $\delta^{\prime}=\delta^{\prime}\left(\epsilon / 2, \Lambda, M_{0}, n\right)>0$ be as in Lemma 3.4 and sufficiently small. Without loss of generality, we can assume that $\delta^{\prime} \leq \epsilon / 2$. Now, let $\delta=\delta\left(\epsilon, \Lambda, M_{0}, n\right):=\delta^{\prime} / C_{1}\left(\Lambda, M_{0}, n\right)$, where $C_{1}\left(\Lambda, M_{0}, n\right) \geq 1$ is some constant which will be determined. Then, choose $\lambda_{0}=\lambda_{0}\left(\delta, M_{1}, \omega_{0}\right)=$ $\lambda_{0}\left(\epsilon, \Lambda, M_{0}, M_{1}, \omega_{0}, n\right)$ sufficiently large such that

$$
\frac{M_{1} K_{\delta}+\delta}{\lambda_{0}} \leq \delta
$$

where $K_{\delta}$ is defined in Lemma 3.3. Now, assume that the assumptions in Proposition 3.2 hold. Let $w$ be as in Lemma 3.3. Then,

$$
\frac{1}{\mu\left(B_{7 r / 8}\right)} \int_{B_{7 r / 8}}|\nabla w|^{2} \mu(x) d x \leq C_{0}(\Lambda) .
$$

Next, let $v$ be as in Lemma 3.4, It follows directly from Lemma 3.4 that

$$
\|\nabla v\|_{L^{\infty}\left(B_{r / 2}\right)} \leq C\left(\Lambda, M_{0}, n\right), \quad \text { and } \quad \frac{1}{\mu\left(B_{r / 2}\right)} \int_{B_{r / 2}}|\nabla w-\nabla v|^{2} \mu(x) d x \leq \frac{\epsilon}{2} .
$$

From this and from Lemma 3.3 , we obtain

$$
\begin{aligned}
& \frac{1}{\mu\left(B_{r / 2}\right)} \int_{B_{r / 2}}|\nabla u-\nabla v|^{2} \mu(x) d x \\
& \leq \frac{1}{\mu\left(B_{r / 2}\right)} \int_{B_{r / 2}}|\nabla u-\nabla w|^{2} \mu(x) d x+\frac{1}{\mu\left(B_{r / 2}\right)} \int_{B_{r / 2}}|\nabla w-\nabla v|^{2} \mu(x) d x \\
& \leq \frac{C(\Lambda) \mu\left(B_{7 r / 8}\right)}{\mu\left(B_{r / 2}\right)}\left[\delta^{2} f_{B_{7 / / 8}}|\nabla u|^{2} d \mu(x)+f_{B_{7 r / 8}}\left|\frac{\mathbf{F}}{\mu}\right|^{2} d \mu(x)+\frac{\left(M_{1} K_{\delta}+\delta\right)^{2}}{\lambda^{2}}\right]+\frac{\epsilon}{2} .
\end{aligned}
$$

This together with 3.9) and the assumptions in the Proposition 3.2 imply

$$
\frac{1}{\mu\left(B_{r / 2}\right)} \int_{B_{r / 2}}|\nabla u-\nabla v|^{2} \mu(x) d x \leq \frac{C(\Lambda) \mu\left(B_{7 r / 8}\right)}{\mu\left(B_{r / 2}\right.} \delta^{2}+\frac{\epsilon}{2}
$$

Moreover, observe that by the doubling property, there is $C\left(M_{0}, n\right)>0$ such that

$$
\frac{\mu\left(B_{7 r / 8}\right)}{\mu\left(B_{r / 2}\right)} \leq C\left(M_{0}, n\right)
$$

Hence, there is $C_{1}\left(\Lambda, M_{0}, n\right) \geq 1$ such that

$$
\frac{1}{\mu\left(B_{r / 2}\right)} \int_{B_{r / 2}}|\nabla u-\nabla v|^{2} \mu(x) d x \leq C_{1}\left(\Lambda, M_{0}, n\right) \delta^{2}+\frac{\epsilon}{2} .
$$

From this and the choice of $\delta$, we obtain

$$
\frac{1}{\mu\left(B_{r / 2}\right)} \int_{B_{r / 2}}|\nabla u-\nabla v|^{2} \mu(x) d x \leq \epsilon,
$$

and the proof is complete.

3.2. Interior level set estimates. This section consists of several lemmas preparing for the proof of Theorem 1.3. For $\lambda>0$, we consider the equation with the scaling parameter $\lambda$

$$
\operatorname{div}\left[\mathbb{A}_{\lambda}(x, u, \nabla u)\right]=\operatorname{div}[\mathbf{F}], \quad \text { in } \quad B_{2 R} .
$$

We begin with the following lemma.

Lemma 3.5. Let $\Lambda>0, M_{0} \geq 1$ be given. There exists $N=N\left(\Lambda, M_{0}, n\right)>1$ such that the following statement holds: Let $M_{1}>0, M_{2} \geq 1, \mathbb{K} \subset \mathbb{R}$ be some open interval, and let $\omega_{0}: \mathbb{K} \times[0, \infty) \rightarrow[0, \infty)$ be continuous satisfying (1.7) and $\left\|\omega_{0}\right\|_{\infty} \leq M_{1}$. Then, for every sufficiently small $\epsilon>0$, there exist sufficiently 
small $\delta=\delta\left(\epsilon, \Lambda, M_{0}, M_{2}, n\right) \in(0,1 / 8)$ and a large number $\lambda_{0}=\lambda_{0}\left(\epsilon, \Lambda, M_{0}, M_{1}, M_{2}, \omega_{0}, n\right) \geq 1$ such that if $\mathbb{A} \in \mathbb{U}_{B_{2 R}, \mathbb{K}}\left(\Lambda, M_{0}, M_{1}, \omega_{0}\right)$ with its asymptotical matrix $\tilde{\mathbb{A}}$ and weight function $\mu \in A_{2}$ satisfying

$$
\sup _{0<\rho \leq R_{0}} \sup _{x \in B_{R}} \frac{1}{\mu\left(B_{\rho}(x)\right)} \int_{B_{\rho}(x)}\left|\tilde{\mathbb{A}}(y)-\langle\tilde{\mathbb{A}}\rangle_{B_{\rho}(x)}\right|^{2} \mu^{-1}(y) d y \leq \delta, \quad \text { for some } R_{0} \in(0, R]
$$

then, for every $\lambda \geq \lambda_{0}$, every $u \in W^{1,2}\left(B_{2 R}, \mu\right)$ a weak solution of $\left(\underline{3.10}\right.$, every $y \in B_{R}$, and every $0<r \leq$ $R_{0} / 3$, if

$$
B_{r}(y) \cap\left\{B_{R}: \mathcal{M}_{\mu, B_{2 R}}\left(|\nabla u|^{2}\right) \leq 1\right\} \cap\left\{B_{R}: \mathcal{M}_{\mu, B_{2 R}}\left(|\mathbf{F} / \mu|^{2}\right) \leq \delta\right\} \neq \emptyset,
$$

then

$$
\omega\left(\left\{x \in B_{R}: \mathcal{M}_{\mu, B_{2 R}}\left(|\nabla u|^{2}\right)>N\right\} \cap B_{r}(y)\right)<\epsilon \omega\left(B_{r}(y)\right),
$$

for every weight function $\omega \in A_{q}$ with $[\omega]_{A_{q}} \leq M_{2}$, for $1 \leq q<\infty$.

Proof. For a given sufficiently small $\epsilon>0$, choose $\gamma \in(0,1)$ sufficiently small, to be determined, and depending only on $\epsilon, \Lambda, M_{0}$ and $M_{2}$. Let $\delta=\delta\left(\gamma, \Lambda, M_{0}, n\right) \in(0,1 / 8)$ and $\lambda_{0}=\lambda_{0}\left(\gamma, M_{0}, M_{1}, M_{2}, \omega_{0}, n\right)$ be defined as in Proposition 3.2. By (3.12), there is $x_{0} \in B_{r}(y)$ such that

$$
\mathcal{M}_{\mu, B_{2 R}}\left(|\nabla u|^{2}\right)\left(x_{0}\right) \leq 1, \quad \text { and } \quad \mathcal{M}_{\mu, B_{2 R}}\left(|\mathbf{F} / \mu|^{2}\right)\left(x_{0}\right) \leq \delta .
$$

Observe that $B_{3 r}(y) \subset B_{2 R}$, and hence $u$ is a weak solution of

$$
\operatorname{div}\left[\mathbb{A}_{\lambda}(x, u, \nabla u)\right]=\operatorname{div}[\mathbf{F}], \quad \text { in } B_{3 r}(y) .
$$

Moreover, since $B_{21 r / 8}(y) \subset B_{29 r / 8}\left(x_{0}\right) \cap B_{2 R}$ and Lemma2.2, we see that

$$
\begin{aligned}
& f_{B_{21 r / 8}(y)}|\nabla u|^{2} d \mu(x) \leq \frac{\mu\left(B_{29 r / 8}\left(x_{0}\right)\right)}{\mu\left(B_{21 r / 8}(y)\right)} \frac{1}{\mu\left(B_{29 r / 8}\left(x_{0}\right)\right)} \int_{B_{29 r / 8}\left(x_{0}\right) \cap B_{2 R}}|\nabla u|^{2} d \mu(x) \leq M_{0}\left(\frac{29}{21}\right)^{2 n}, \\
& f_{B_{21 r / 8}(y)}|\mathbf{F} / \mu|^{2} d \mu(x) \leq \frac{\mu\left(B_{29 r / 8}\left(x_{0}\right)\right)}{\mu\left(B_{21 r / 8}(y)\right)} \frac{1}{\mu\left(B_{29 r / 8}\left(x_{0}\right)\right)} \int_{B_{29 r / 8}\left(x_{0}\right) \cap B_{2 R}}|\mathbf{F} / \mu|^{2} d \mu(x) \leq M_{0}\left(\frac{29}{21}\right)^{2 n} \delta .
\end{aligned}
$$

Moreover, because $3 r \leq R_{0}$, with the assumption (3.11) of the lemma, we see that

$$
\frac{1}{\mu\left(B_{3 r}(y)\right)} \int_{B_{3 r}(y)}\left|\tilde{\mathbb{A}}(x)-\langle\tilde{\mathbb{A}}\rangle_{B_{3 r}(y)}\right|^{2} \mu^{-1}(x) d x \leq \delta .
$$

Now, let $u^{\prime}(x)=u(x) /\left[M_{0}\left(\frac{29}{21}\right)^{2 n}\right], \mathbf{F}^{\prime}(x)=\mathbf{F}(x) /\left[M_{0}\left(\frac{29}{21}\right)^{2 n}\right]$, and $\lambda^{\prime}=\lambda M_{0}\left(\frac{29}{21}\right)^{2 n} \geq \lambda_{0}$, we infer from (3.14) that $u^{\prime}$ is a weak solution of

$$
\operatorname{div}\left[\mathbb{A}_{\lambda^{\prime}}\left(x, u^{\prime}, \nabla u^{\prime}\right]=\operatorname{div}\left[\mathbf{F}^{\prime}(x)\right], \quad x \in B_{3 r}(y) .\right.
$$

Therefore, by applying Proposition 3.2 for $u^{\prime}, F^{\prime}$ and scaling back to $u, F$, we see that there exists $v$ such that

$$
f_{B_{3 r / 2}(y)}|\nabla u-\nabla v|^{2} d \mu(x) \leq \gamma M_{0}\left(\frac{29}{21}\right)^{2 n}, \quad\|\nabla v\|_{L^{\infty}\left(B_{3 r / 2}(y)\right)} \leq C_{*}\left(\Lambda, M_{0}, n\right) .
$$

Now, let

$$
N=\max \left\{4 C_{*}\left(\Lambda, M_{0}, n\right), 5^{2 n} M_{0}\right\}
$$

We claim that

$$
\left\{B_{r}(y): \mathcal{M}_{\mu, B_{3 r / 2}(y)}\left(|\nabla u-\nabla v|^{2}\right) \leq C_{*}\right\} \subset\left\{B_{r}(y): \mathcal{M}_{\mu, B_{2 R}}\left(|\nabla u|^{2}\right) \leq N\right\} .
$$

Indeed, let $x$ to be any point in the set on the left hand side of (3.16). We only need to show that

$$
\mathcal{M}_{\mu, B_{2 R}}\left(|\nabla u|^{2}\right)(x) \leq N .
$$


Consider the ball $B_{\rho}(x)$. If $\rho \leq r / 2$, we see that $B_{\rho}(x) \subset B_{3 r / 2}(y) \subset B_{2 R}$. From this, it follows

$$
\begin{aligned}
\frac{1}{\mu\left(B_{\rho}(x)\right)} \int_{B_{\rho}(x)}|\nabla u|^{2} \mu(x) d x & \leq 2\left[\frac{1}{\mu\left(B_{\rho}(x)\right)} \int_{B_{\rho}(x)}|\nabla u-\nabla v|^{2} \mu(x) d x+\frac{1}{\mu\left(B_{\rho}(x)\right)} \int_{B_{\rho}(x)}|\nabla v|^{2} \mu(x) d x\right] \\
& \leq 2\left[\mathcal{M}_{\mu, B_{3 r / 2}(y)}\left(|\nabla u-\nabla v|^{2}\right)(x)+\|\nabla v\|_{L^{\infty}\left(B_{3 r / 2}(y)\right.}\right] \\
& \leq 2\left[C_{*}+C_{*}\right]=4 C_{*} \leq N .
\end{aligned}
$$

On the other hand, if $\rho>r / 2$, we see that $B_{\rho}(x) \subset B_{5 \rho}\left(x_{0}\right)$. Hence, by applying Lemma2.2 -(i), and using (3.13), we obtain

$$
\frac{1}{\mu\left(B_{\rho}(x)\right)} \int_{B_{\rho}(x) \cap B_{2 R}}|\nabla u|^{2} \mu(x) d x \leq \frac{\mu\left(B_{5 \rho}\left(x_{0}\right)\right)}{\mu\left(B_{\rho}(x)\right)} \frac{1}{\mu\left(B_{5 \rho}\left(x_{0}\right)\right)} \int_{B_{5 \rho}\left(x_{0}\right) \cap B_{2 R}}|\nabla u|^{2} \mu(x) d x \leq M_{0} 5^{2 n} \leq N .
$$

We therefore have proved (3.17), which in turns proves (3.16). Observe that (3.16) is equivalent to

$$
\left\{B_{r}(y): \mathcal{M}_{\mu, B_{2 R}}\left(|\nabla u|^{2}\right)>N\right\} \subset E:=\left\{B_{r}(y): \mathcal{M}_{\mu, B_{3 r / 2}(y)}\left(|\nabla u-\nabla v|^{2}\right)>C_{*}\right\} .
$$

On one hand, by the weak type $(1,1)$ estimate, the doubling property of $\mu$ in Lemma 2.2, and (3.15), we see that

$$
\mu(E) \leq \frac{C\left(M_{0}\right) \mu\left(B_{3 r / 2}(y)\right)}{C_{*}} f_{B_{3 r / 2}(y)}|\nabla u-\nabla v|^{2} d \mu(x) \leq C^{\prime}\left(\Lambda, M_{0}, n\right) \mu\left(B_{r}(y)\right) \gamma .
$$

In other words,

This and Lemma 2.3 imply

$$
\frac{\mu(E)}{\mu\left(B_{r}(y)\right)} \leq C^{\prime}\left(\Lambda, M_{0}, n\right) \gamma
$$

$$
\frac{\omega(E)}{\omega\left(B_{r}(y)\right)} \leq C\left(M_{0}, M_{2}\right)\left(\frac{\mu(E)}{\mu\left(B_{r}(y)\right)}\right)^{\beta / 2} \leq C^{*}\left(\Lambda, M_{0}, M_{2}, n\right) \gamma^{\beta / 2}
$$

for some constant $\beta=\beta\left(M_{2}, n\right)>0$. Hence, it follows from this and (3.18) that

$$
\omega\left(\left\{B_{r}(y): \mathcal{M}_{\mu, B_{2 R}}\left(|\nabla u|^{2}\right)>N\right\}\right) \leq \omega(E) \leq C^{*}\left(\Lambda, M_{0}, M_{2}, n\right) \gamma^{\beta / 2} \omega\left(B_{r}(y)\right) .
$$

Now, if we choose $\gamma$ such that $\gamma^{\beta / 2} C^{*}\left(\Lambda, M_{0}, M_{2}, n\right)=\epsilon$, the lemma follows.

We now can estimate a level set of $\mathcal{M}_{\mu, B_{2 R}}\left(|\nabla u|^{2}\right)$. This is the main result in this subsection.

Proposition 3.6. Let $\Lambda>0, M_{k}$, and $\omega_{0}, N$ be as in Lemma 3.5 with $k=1,2,3$. For any $\epsilon>0$, let $\delta \in(0,1 / 8)$ and $\lambda_{0} \geq 1$ be defined as in Lemma 3.5 Suppose that $\mathbb{A} \in \mathbb{U}_{B_{2 R}, \mathbb{K}}\left(\Lambda, M_{0}, M_{1}, \omega_{0}\right)$ with its asymptotical matrix $\tilde{\mathbb{A}}$ and weight $\mu$ satisfying

$$
\sup _{0<\rho \leq R_{0}} \sup _{x \in B_{R}} \frac{1}{\mu\left(B_{\rho}(x)\right)} \int_{B_{\rho}(x)}\left|\tilde{\mathbb{A}}(y)-\langle\tilde{\mathbb{A}}\rangle_{B_{\rho}(x)}\right|^{2} \mu^{-1}(y) d y \leq \delta, \quad \text { for some } \quad R_{0} \in(0, R] .
$$

Then, for every $\lambda \geq \lambda_{0}$ for $\omega \in A_{q}$ with $[\omega]_{A_{q}} \leq M_{2}, 1<q<\infty$ and for every $u \in W^{1,2}\left(B_{2 R}, \mu\right)$ a weak solution of (3.10), if

$$
\omega\left(\left\{B_{R}: \mathcal{M}_{\mu, B_{2 R}}\left(|\nabla u|^{2}\right)>N\right\}\right) \leq \epsilon \omega\left(B_{R_{0} / 6}(y)\right), \quad \forall y \in B_{R},
$$

then for $\epsilon_{1}=(20)^{q n} M_{2}^{2} \epsilon$,

$$
\omega\left(\left\{B_{R}: \mathcal{M}_{\mu, B_{2 R}}\left(|\nabla u|^{2}\right)>N\right\}\right) \leq \epsilon_{1}\left[\omega\left(\left\{B_{R}: \mathcal{M}_{\mu, B_{2 R}}\left(|\nabla u|^{2}\right)>1\right\}\right)+\omega\left(\left\{B_{R}: \mathcal{M}_{\mu, B_{2 R}}\left(|\mathbf{F} / \mu|^{2}\right)>\delta\right\}\right)\right] .
$$

Proof. Let us denote

$$
C=\left\{B_{R}: \mathcal{M}_{\mu, B_{2 R}}\left(|\nabla u|^{2}\right)>N\right\}
$$

and

$$
D=\left\{B_{R}: \mathcal{M}_{\mu, B_{2 R}}\left(|\nabla u|^{2}\right)>1\right\} \cup\left\{B_{R}: \mathcal{M}_{\mu, B_{R}}\left(|\mathbf{F} / \mu|^{2}\right)>\delta\right\}
$$


Clearly, $C \subset D \subset B_{R}$. Our goal is to apply the modified Vitali's covering, Lemma 2.8, with $r_{0}=R_{0} / 6$. Clearly by the assumption of the lemma, $\omega(C) \leq \epsilon \omega\left(B_{r_{0}}(y)\right)$ forall $y \in B_{R}$. Moreover, for some $\rho \in\left(0, r_{0}\right)$ and $y \in B_{R}$, if $\omega\left(C \cap B_{\rho}(y)\right) \geq \epsilon \omega\left(B_{\rho}(y)\right)$, then it follows from Lemma 3.5 that

$$
B_{\rho}(y) \cap B_{R} \subset D .
$$

This verifies all conditions in the modified Vitali's covering lemma, Lemma 2.8. Therefore, Lemma 3.6 follows.

3.3. Proof of Theorem 1.3, By iterating Proposition 3.6, we obtain the following result.

Lemma 3.7. Let $\Lambda>0, M_{k}$, and $\omega_{0}, N$ be as in Lemma 3.5 with $k=1,2,3$. For any $\epsilon>0$, let $\delta \in$ $(0,1 / 8), \lambda_{0} \geq 1$ be defined as in Lemma 3.5. Suppose that $\mathbb{A} \in \mathbb{U}_{B_{2 R}, \mathbb{K}}\left(\Lambda, M_{0}, M_{1}, \omega_{0}\right)$ with its asymptotical matrix $\tilde{\mathbb{A}}$ and weight $\mu$ satisfying

$$
\sup _{0<\rho \leq R_{0}} \sup _{x \in B_{R}} \frac{1}{\mu\left(B_{\rho}(x)\right)} \int_{B_{\rho}(x)}\left|\tilde{\mathbb{A}}(y)-\langle\tilde{\mathbb{A}}\rangle_{B_{\rho}(x)}\right|^{2} \mu^{-1}(y) d y \leq \delta, \quad \text { for some } \quad R_{0} \in(0, R] .
$$

Then, for every $\lambda \geq \lambda_{0}$ for $\omega \in A_{q}$ with $[\omega]_{A_{q}} \leq M_{2}$ with $1<q<\infty$, and for every $u \in W^{1,2}\left(B_{2 R}, \mu\right)$ a weak solution of (3.10), if

$$
\omega\left(\left\{B_{R}: \mathcal{M}_{\mu, B_{2 R}}\left(|\nabla u|^{2}\right)>N\right\}\right) \leq \epsilon \omega\left(B_{R_{0} / 6}(y)\right), \quad \forall y \in B_{R},
$$

then for $\epsilon_{1}=(20)^{q n} M_{2}^{2} \epsilon$,

$$
\begin{aligned}
\omega\left(\left\{B_{R}: \mathcal{M}_{\mu, B_{2 R}}\left(|\nabla u|^{2}\right)>N^{k}\right\}\right) \leq & \epsilon_{1}^{k} \omega\left(\left\{B_{R}: \mathcal{M}_{\mu, B_{2 R}}\left(|\nabla u|^{2}\right)>1\right\}\right) \\
& +\sum_{i=1}^{k} \epsilon_{1}^{i} \omega\left(\left\{B_{R}: \mathcal{M}_{\mu, B_{2 R}}\left(|\mathbf{F} / \mu|^{2}\right)>\delta N^{k-i}\right\}\right) .
\end{aligned}
$$

Proof. We skip the proof because it is the same as that of Lemma4.10 below.

After the accomplishment of Lemma 3.7, the rest of the proof of Theorem 1.3 is similar to that of Theorem 1.6 in the next section. We therefore skip it.

\section{LOCAL BOUNDARY $W^{1, p}$-REGULARITY THEORY AND GLOBAL $W^{1, p}$-REGULARITY THEORY}

To establish the global regularity estimates, we need both interior estimates and estimates up to the boundary. The interior theory are accomplished in previous sections. We now need to create the same theory up to the boundary.

4.1. Boundary approximation estimates. For $r>0$ and for $x_{0}=\left(x_{1}^{0}, x_{2}^{0}, \cdots, x_{n}^{0}\right) \in \mathbb{R}^{n}$, let us denote

$$
\begin{aligned}
& B_{r}^{+}\left(x_{0}\right)=\left\{y=\left(y_{1}, y_{2}, \cdots, y_{n}\right) \in B_{r}\left(x_{0}\right): y_{n}>x_{n}^{0}\right\}, \quad B_{r}^{+}=B_{r}^{+}(0), \\
& T_{r}\left(x_{0}\right)=\left\{x=\left(x_{1}, x_{2}, \cdots, x_{n}\right) \in \partial B_{r}^{+}(0): x_{n}=x_{n}^{0}\right\}, \quad T_{r}=T_{r}(0) \text {. }
\end{aligned}
$$

For given $x_{0} \in \mathbb{R}^{n}$ and $\Omega \subset \mathbb{R}^{n}$, we also denote

$$
\Omega_{r}\left(x_{0}\right)=\Omega \cap B_{r}\left(x_{0}\right), \quad \partial_{w} \Omega_{r}\left(x_{0}\right)=\partial \Omega \cap B_{r}\left(x_{0}\right), \quad \Omega_{r}=\Omega_{r}(0) .
$$

For your convenience, let us mention that the $\left(\delta, R_{0}\right)$-Reifenberg flat domains are defined in Definition 1.4 In this section, for $r>0, \lambda>0$, and $\mathbb{A} \in \mathbb{U}_{\Omega_{r}, \mathbb{K}}\left(\Lambda, M_{0}, M_{1}, \omega_{0}\right)$, with some given $\omega_{0}$, we study the problem

$$
\left\{\begin{array}{ccccc}
\operatorname{div}\left[\mathbb{A}_{\lambda}(x, u, \nabla u)\right] & = & \operatorname{div}[\mathbf{F}] & \text { in } & \Omega_{r}, \\
u & = & g & \text { on } \partial_{w} \Omega_{r} .
\end{array}\right.
$$

The main result of the subsection is the following Proposition 
Proposition 4.1. Let $\Lambda>0, M_{0} \geq 1$, and $M_{1}>0$. Then, for every sufficiently $\epsilon>0$, there are $\delta=$ $\delta\left(\epsilon, \Lambda, M_{0}, n\right)>0$ sufficiently small and $\lambda_{0}=\lambda_{0}\left(\epsilon, \Lambda, M_{0}, M_{1}, \omega_{0}, n\right) \geq 1$ such that the following statement holds true: Assume that $\mathbb{A} \in \mathbb{U}_{\Omega_{r}, \mathbb{K}}\left(\Lambda, M_{0}, M_{1}, \omega_{0}\right)$, for some $r>0$, some open interval $\mathbb{K} \subset \mathbb{R}$, and with its corresponding asymptotical matrix $\tilde{\mathbb{A}}$, and weight $\mu \in A_{2}$. Assume also that

$$
B_{r}^{+} \subset \Omega_{r} \subset B_{r} \cap\left\{x_{n}>-4 r \delta\right\}, \quad \frac{1}{\mu\left(B_{r}\right)} \int_{\Omega_{r}}\left|\tilde{\mathbb{A}}(x)-\langle\tilde{\mathbb{A}}\rangle_{\Omega_{r}}\right|^{2} \mu^{-1}(x) d x \leq \delta,
$$

and

$$
\frac{1}{\mu\left(B_{7 r / 8}\right)} \int_{\Omega_{7 r / 8}}\left|\frac{\mathbf{F}}{\mu}\right|^{2} \mu(x) d x \leq \delta, \quad \frac{1}{\mu\left(B_{7 r / 8}\right)} \int_{\Omega_{7 r / 8}}|\nabla g|^{2} \mu(x) d x \leq \delta,
$$

and if $\lambda \geq \lambda_{0}$, and $u \in W^{1,2}\left(\Omega_{r}, \mu\right)$ is a weak solution of (4.1) satisfying

$$
\frac{1}{\mu\left(B_{7 r / 8}\right)} \int_{\Omega_{7 r / 8}}|\nabla u|^{2} \mu(x) \leq 1,
$$

then, there is a function $v$ defined in $\Omega_{3 r / 4}$ and a constant $C=C\left(\Lambda, M_{0}, n\right)$ such that

$$
\frac{1}{\mu\left(B_{r / 2}\right)} \int_{\Omega_{r / 2}}|\nabla u-\nabla v|^{2} \mu(x) d x \leq \epsilon, \quad \text { and } \quad\|\nabla v\|_{L^{\infty}\left(\Omega_{r / 2}\right)} \leq C .
$$

The remaining of the section is to prove Proposition 4.1. The proof is divided into two steps of approximations, Lemma 4.4 and Lemma 4.5 below.

4.1.1. Step 1: First approximation. We write $\theta=\frac{7 r}{8}$. We first approximate the solution $u$ of (4.1) by and the corresponding homogeneous equation

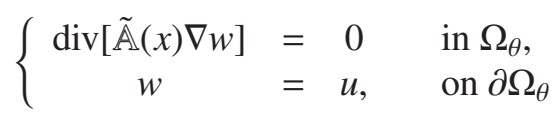

We now state our definition of weak solutions for (4.1), meanwhile weak solutions for (4.2) are understood by Definition 1.2 .

Definition 4.2. For $g \in W^{1,2}\left(\Omega_{r}, \mu\right)$, and $\mathbf{F} \in L^{2}\left(\Omega_{r}, \mu^{-1}\right)$, a function $u \in W^{1,2}\left(\Omega_{r}, \mu\right)$ is a weak solution to (4.1) if $u-g \in \stackrel{o}{W}^{1,2}\left(\Omega_{r}, \mu\right), u(x) \in \mathbb{K}_{\lambda}:=\mathbb{K} / \lambda$ for a.e. $x \in \Omega_{r}$, and

$$
\int_{\Omega_{r}}\left\langle\mathbb{A}_{\lambda}(x, u, \nabla u), \nabla \varphi\right\rangle d x=\int_{\Omega_{r}}\langle\mathbf{F}, \nabla \varphi\rangle d x, \quad \forall \varphi \in C_{0}^{\infty}\left(\Omega_{r}\right)
$$

where $\stackrel{o}{W}^{1,2}\left(\Omega_{r}, \mu\right)$ denotes all functions $f \in W^{1,2}\left(\Omega_{r}, \mu\right)$ so that its zero extension to $B_{r}$ are in $W^{1,2}\left(B_{r}, \mu\right)$.

Remark 4.3. Given $u \in W^{1,2}\left(\Omega_{r}, \mu\right)$, it can be shown that $w$ is a weak solution of (4.2) if and only if $\tilde{w}=w-u \in W_{0}^{1,2}\left(\Omega_{\theta}, \mu\right)$ is a weak solution of

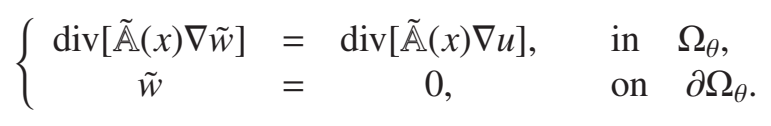

For the later, its existence and uniqueness of the weak solution $\tilde{w}$ is established in [15, Theorem 2.2].

The following energy estimates are the main result of our first approximation step.

Lemma 4.4. Assume that $u \in W^{1,2}\left(\Omega_{r}, \mu\right)$ is a weak solution of (4.1) and $w \in W^{1,2}\left(\Omega_{\theta}, \mu\right)$ is a weak solution of 4.2). Then, there is $C=C(\Lambda)>0$ such that

$$
\int_{\Omega_{\theta}}|\nabla w|^{2} \mu(x) d x \leq C(\Lambda) \int_{\Omega_{\theta}}|\nabla u|^{2} \mu(x) d x .
$$


Moreover, for every $\delta>0$, there is $K_{\delta}>0$ depending on $\delta$ and $\omega_{0}$ such that

$$
\begin{aligned}
& \frac{1}{\mu\left(B_{\theta}\right)} \int_{\Omega_{\theta}}|\nabla(u-w)|^{2} \mu(x) d x \\
& \leq C\left(\Lambda, M_{0}\right)\left[\frac{\delta^{2}}{\mu\left(B_{\theta}\right)} \int_{\Omega_{\theta}}|\nabla u|^{2} \mu(x) d x+\frac{1}{\mu\left(B_{\theta}\right)} \int_{\Omega_{\theta}}\left|\frac{\mathbf{F}}{\mu}\right|^{2} \mu(x) d x+\frac{M_{1}^{2} K_{\delta}^{2}+\delta^{2}}{\lambda^{2}}\right] .
\end{aligned}
$$

Proof. Since $w-u \in W_{0}^{1,2}\left(\Omega_{\theta}, \mu\right)$, we can use it as a test function for the equation (4.2) to obtain

$$
\int_{\Omega_{\theta}}\langle\tilde{\mathbb{A}}(x) \nabla w, \nabla w\rangle d x=\int_{\Omega_{\theta}}\langle\tilde{\mathbb{A}}(x) \nabla w, \nabla u\rangle d x .
$$

From this, (1.8), and the Hölder's inequality, we obtain

$$
\Lambda^{-1} \int_{\Omega_{\theta}}|\nabla w|^{2} \mu(x) d x \leq \Lambda\left\{\int_{\Omega_{\theta}}|\nabla u|^{2} \mu(x) d x\right\}^{1 / 2}\left\{\int_{\Omega_{\theta}}|\nabla u|^{2} \mu d x\right\}^{1 / 2} .
$$

Hence

$$
\int_{\Omega_{\theta}}|\nabla w|^{2} \mu(x) d x \leq C(\Lambda) \int_{\Omega_{\theta}}|\nabla u|^{2} \mu(x) d x
$$

Therefore, we obtain (4.3). We next prove (4.4). Observe that it follows from (4.5) that

$$
\int_{\Omega_{\theta}}\langle\tilde{\mathbb{A}}(x) \nabla(u-w), \nabla(u-w)\rangle d x=\int_{\Omega_{\theta}}\langle\tilde{\mathbb{A}}(x) \nabla u, \nabla(u-w)\rangle d x .
$$

On the other hand, use $u-w \in W^{1,2}\left(\Omega_{\theta}, \mu\right)$ as a test function for the equation (4.1), we see that

$$
\int_{\Omega_{\theta}}\left\langle\mathbb{A}_{\lambda}(x, u, \nabla u), \nabla(u-w)\right\rangle d x=\int_{\Omega_{\theta}}\langle\mathbf{F}, \nabla(u-w)\rangle d x .
$$

Combing the last two identity, we obtain

$$
\int_{\Omega_{\theta}}\langle\tilde{\mathbb{A}}(x) \nabla(u-w), \nabla(u-w)\rangle d x=\int_{\Omega_{\theta}}\left\langle\tilde{\mathbb{A}}(x) \nabla u-\mathbb{A}_{\lambda}(x, u, \nabla u), \nabla(u-w)\right\rangle d x+\int_{\Omega_{\theta}}\langle\mathbf{F}, \nabla(u-w)\rangle d x .
$$

The proof now follows exactly the same as that of Lemma 3.3 with the note that $\mu\left(B_{\theta}\right) \sim \mu\left(\Omega_{\theta}\right)$. We then skip it.

4.1.2. Step 2: Second approximation. We approximate the weak solution $w$ of (4.2) by the solution $v$ of the following equation on the flat domain

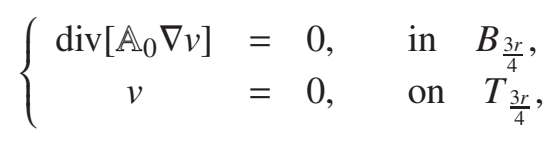

for some constant, symmetric, uniformly elliptic matrix that is sufficiently close to $\langle\tilde{\mathbb{A}}\rangle_{\Omega_{r}}$. Our next lemma is the main result of this subsection.

Lemma 4.5. Let $\Lambda>0, M_{0} \geq 1$ be fixed. For every $\epsilon>0$ sufficiently small, there exists sufficiently small number $\delta^{\prime}>0$ depending on only $\epsilon, \Lambda, n$, and $M_{0}$ such that for every $\delta \in\left(0, \delta^{\prime}\right]$, the following statement holds true: If (1.8) holds, $[\mu]_{A_{2}} \leq M_{0}$,

$$
\frac{1}{\mu\left(B_{r}\right)} \int_{\Omega_{r}}\left|\tilde{\mathbb{A}}-\langle\tilde{\mathbb{A}}\rangle_{\Omega_{r}}\right|^{2} \mu^{-1} d x \leq \delta^{2}, \quad \frac{1}{\mu\left(B_{7 r / 8}\right)} \int_{\Omega_{7_{r / 8}}}|\nabla g|^{2} \mu(x) d x \leq \delta^{2},
$$

and if $w$ is a weak solution of

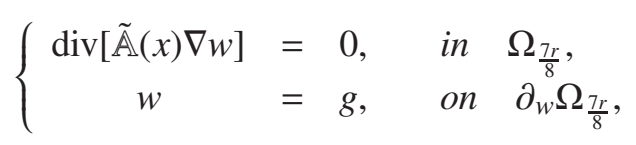


satisfying

$$
\frac{1}{\mu\left(\Omega_{\frac{7 r}{8}}\right)} \int_{\Omega_{\frac{7 r}{8}}}|\nabla w|^{2} d \mu \leq C_{0}, \quad \text { with some } \quad C_{0}=C_{0}\left(\Lambda, M_{0}\right) \geq 1,
$$

then there exists a constant, symmetric matrix $\mathbb{A}_{0}$ and a weak solution $v$ of (4.6) satisfying

$$
\left|\langle\tilde{\mathbb{A}}\rangle_{\Omega_{r}}-\mathbb{A}_{0}\right| \leq \frac{\epsilon \mu\left(B_{r}\right)}{\left|B_{r}\right|}, \text { and } \frac{1}{\mu\left(B_{r / 2}\right)} \int_{\Omega_{r / 2}}|\nabla w-\nabla v|^{2} \mu(x) d x \leq \epsilon .
$$

Moreover, there is $C=C\left(\Lambda, n, M_{0}\right)$ such that

$$
\|\nabla v\|_{L^{\infty}\left(\Omega_{r / 2}\right)} \leq C\left(\Lambda, n, M_{0}\right) .
$$

Proof. Let us denote $g^{\prime}=\frac{g}{C_{0}}, w^{\prime}=\frac{w-g}{C_{0}}$, and $\mathbf{F}^{\prime}(x)=\tilde{\mathbb{A}}(x) \nabla g^{\prime}(x)$. Then, $w^{\prime} \in W^{1,2}\left(\Omega_{\frac{7 r}{8}}, \mu\right)$ is a weak solution of

Observe that

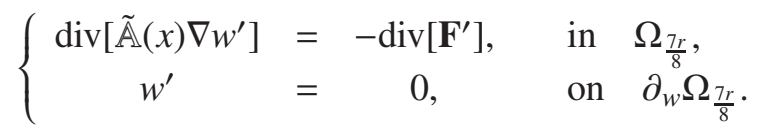

$$
\frac{1}{\mu\left(B_{7 r / 8}\right)} \int_{\Omega_{7 r / 8}}\left|\frac{\mathbf{F}^{\prime}}{\mu}\right|^{2} \mu(x) d x=\frac{1}{\mu\left(B_{7 r / 8}\right)} \int_{\Omega_{7 r / 8}}\left|\frac{\tilde{\mathbb{A}} \nabla g^{\prime}}{\mu(x)}\right|^{2} \mu(x) \leq \frac{\Lambda^{2}}{\mu\left(B_{7 r / 8}\right)} \int_{\Omega_{7 r / 8}}\left|\nabla g^{\prime}\right|^{2} \mu(x) d x \leq \Lambda^{2} \delta .
$$

Moreover,

$$
\frac{1}{\mu\left(B_{7 r / 8}\right)} \int_{\Omega_{7 r / 8}}\left|\nabla w^{\prime}\right|^{2} \mu(x) d x \leq 1 .
$$

The result then follows from [8, Proposition 5.5] by a suitable scaling.

4.1.3. Proof of Proposition 4.1 We skip it because it is the same as that of Proposition 3.2

4.2. Level set estimates up to the boundary. To obtain the estimates of level sets for the maximal function $\mathcal{M}_{\mu}\left(|\nabla u|^{2} \chi_{\Omega}\right)$, we consider the following equation with the scaling parameter $\lambda>0$ :

$$
\left\{\begin{array}{ccccc}
\operatorname{div}\left[\mathbb{A}_{\lambda}(x, u, \nabla u)\right] & = & \operatorname{div}[\mathbf{F}], & & \text { in } \Omega . \\
u & = & g, & & \text { on } \partial \Omega .
\end{array}\right.
$$

We need several intermediate steps in order the prove the Proposition 4.9 below, which is the main result of the subsection. Our first lemma is in the same fashion as Lemma 3.5

Lemma 4.6. Let $\Lambda>0, M_{0} \geq 1$ be given. There exists $N_{1}=N_{1}\left(\Lambda, M_{0}, n\right)>1$ such that the following statement holds: Let $M_{1}>0, M_{2} \geq 1, \mathbb{K} \subset \mathbb{R}$ be some open interval, and let $\omega_{0}: \mathbb{K} \times[0, \infty) \rightarrow[0, \infty)$ be continuous satisfying (1.7) and $\left\|\omega_{0}\right\|_{\infty} \leq M_{1}$. Then, for every sufficiently small $\epsilon>0$, there exist sufficiently small $\delta_{1}=\delta_{1}\left(\epsilon, \Lambda, M_{0}, M_{2}, n\right)>0$ and a large number $\lambda_{1}=\lambda_{1}\left(\epsilon, \Lambda, M_{0}, M_{1}, M_{2}, \omega_{0}, n\right) \geq 1$ such that for every $R>0, y_{0} \in \bar{\Omega}$ if $0 \in \Omega_{R}\left(y_{0}\right)$, and if $\mathbb{A} \in \mathbb{U}_{\Omega, \mathbb{K}}\left(\Lambda, M_{0}, M_{1}, \omega_{0}\right)$ with its asymptotical matrix $\tilde{\mathbb{A}}$ and weight function $\mu \in A_{2}$ satisfying

$$
\frac{1}{\mu\left(B_{3 r}\right)} \int_{\Omega_{3 r}}\left|\tilde{\mathbb{A}}(z)-\langle\tilde{\mathbb{A}}\rangle_{\Omega_{3 r}(0)}\right|^{2} \mu^{-1}(z) d z \leq \delta_{1}
$$

for some $r \in(0, R / 4)$, and

$$
B_{3 r}^{+}(0) \subset \Omega_{3 r}(0) \subset B_{3 r}(0) \cap\left\{\left(x^{\prime}, x_{n}\right) \in \mathbb{R}^{n}: x_{n}>-12 r \delta_{1}\right\}
$$

then for every $\lambda \geq \lambda_{1}$ and every weak solution $u \in W^{1,2}(\Omega, \mu)$ of (4.9) satisfying

then it holds

$$
\begin{aligned}
B_{r} & \cap\left\{\Omega_{R}\left(y_{0}\right): \mathcal{M}_{\mu, \Omega_{2 R}\left(y_{0}\right)}\left(|\nabla u|^{2}\right) \leq 1\right\} \cap \\
& \cap\left\{\Omega_{R}\left(y_{0}\right): \mathcal{M}_{\mu, \Omega_{2 R}\left(y_{0}\right)}\left(|\mathbf{F} / \mu|^{2}\right) \leq \delta_{1}\right\} \cap\left\{\Omega_{R}\left(y_{0}\right): \mathcal{M}_{\mu, \Omega_{2 R}\left(y_{0}\right)}\left(|\nabla g|^{2}\right) \leq \delta_{1}\right\} \neq \emptyset,
\end{aligned}
$$

$$
\omega\left(\left\{x \in \Omega_{R}\left(y_{0}\right): \mathcal{M}_{\mu, \Omega_{2 R}\left(y_{0}\right)}\left(|\nabla u|^{2}\right)>N_{1}\right\} \cap B_{r}\right)<\epsilon \omega\left(B_{r}\right),
$$

for every weight function $\omega \in A_{q}$ with $[\omega]_{A_{q}} \leq M_{2}$, for $1 \leq q<\infty$. 
Proof. Though similar Lemma 3.5, but details are different, hence a proof is needed. Let $\gamma>0$ sufficiently small to be determined and depending on $\epsilon, \Lambda, M_{0}, M_{2}, n$. Let $\delta_{1}=\delta\left(\gamma, \Lambda, M_{0}, n\right)>0$ and sufficiently small, and $\lambda_{1}=\lambda_{0}\left(\gamma, M_{0}, M_{1}, \omega_{0}, n\right) \geq 1$, where $\delta$ and $\lambda_{0}$ are defined in Proposition 4.1 By (4.10), there is $x_{0} \in \Omega \cap B_{r} \cap B_{R}\left(y_{0}\right)$ such that

$$
\left.\mathcal{M}_{\mu, \Omega_{2 R}\left(y_{0}\right)}\left(|\nabla u|^{2}\right)\left(x_{0}\right) \leq 1, \quad \mathcal{M}_{\mu, \Omega_{2 R}\left(y_{0}\right)}(\mid \mathbf{F} / \mu)\right)\left(x_{0}\right) \leq \delta_{1}, \quad \mathcal{M}_{\mu, \Omega_{2 R}\left(y_{0}\right)}\left(|\nabla g|^{2}\right)\left(x_{0}\right) \leq \delta_{1} .
$$

From this and since $\Omega_{21 r / 8}=B_{21 r / 8} \cap \Omega \subset \Omega_{29 r / 8}\left(x_{0}\right)=B_{29 r / 8}\left(x_{0}\right) \cap \Omega \subset \Omega_{2 R}\left(y_{0}\right)$, we obtain

$$
\begin{gathered}
\frac{1}{\mu\left(B_{21 r / 8}\right)} \int_{\Omega_{21 r / 8}}|\nabla u|^{2} \mu(x) d x \leq \frac{\mu\left(B_{29 r / 8}\left(x_{0}\right)\right)}{\mu\left(B_{29 r / 8}\right)} \frac{1}{\mu\left(B_{29 r / 8}\left(x_{0}\right)\right)} \int_{\Omega_{29 r / 8}\left(x_{0}\right)}|\nabla u|^{2} \mu(x) d x \leq M_{0}(29 / 21)^{2 n}, \\
\frac{1}{\mu\left(B_{21 r / 8}\right)} \int_{\Omega_{21 r / 8}}|\mathbf{F} / \mu|^{2} \mu(x) d x \leq \frac{\mu\left(B_{29 r / 8}\left(x_{0}\right)\right)}{\mu\left(B_{21 r / 8}\right)} \frac{1}{\mu\left(B_{29 r / 8}\left(x_{0}\right)\right)} \int_{\Omega_{29 r / 8}\left(x_{0}\right)}|\mathbf{F} / \mu|^{2} \mu(x) d x \leq M_{0}(29 / 21)^{2 n} \delta_{1}, \\
\frac{1}{\mu\left(B_{21 r / 8}\right)} \int_{\Omega_{21 r / 8}}|\nabla g|^{2} \mu(x) d x \leq \frac{\mu\left(B_{29 r / 8}\left(x_{0}\right)\right)}{\mu\left(B_{21 r / 8}\right)} \frac{1}{\mu\left(B_{29 r / 8}\left(x_{0}\right)\right)} \int_{\Omega_{29 r / 8}\left(x_{0}\right)}|\nabla g|^{2} \mu(x) d x \leq M_{0}(29 / 21)^{2 n} \delta_{1},
\end{gathered}
$$

Then, with suitable scaling, we can apply Proposition 4.1 to find a function $v$ such that

$$
\frac{1}{\mu\left(B_{3 r / 2}\right)} \int_{\Omega_{3 r / 2}}|\nabla u-\nabla v|^{2} \mu(x) d x \leq \gamma M_{0}(29 / 21)^{2 n}, \quad\|\nabla v\|_{L^{\infty}\left(\Omega_{3 r / 2}\right)} \leq C_{*}=C\left(\Lambda, M_{0}, n\right) .
$$

Let $N_{1}=\max \left\{4 C_{*}, 5^{2 n} M_{0}\right\}$, we claim that

$$
\left\{x \in \Omega_{r}: \mathcal{M}_{\mu, \Omega_{3 r / 2}}\left(|\nabla u-\nabla v|^{2}\right) \leq C_{*}\right\} \subset\left\{x \in \Omega_{r}: \mathcal{M}_{\mu, \Omega_{2 R}\left(y_{0}\right)}\left(|\nabla u|^{2}\right) \leq N_{1}\right\} .
$$

Indeed, let $x$ be a point in the set of the left hand side of [4.13), we need to show that

$$
\mathcal{M}_{\mu, \Omega_{2 R}\left(y_{0}\right)}\left(|\nabla u|^{2}\right)(x) \leq N_{1} \text {. }
$$

For $\rho>0$, and consider the case $\rho<r / 2$. In this case, $\Omega_{\rho}(x)=\Omega \cap B_{\rho}(x) \subset \Omega_{3 r / 2}=\Omega \cap B_{3 r / 2}(0) \subset \Omega_{2 R}\left(y_{0}\right)$. Therefore,

$$
\begin{aligned}
\frac{1}{\mu\left(B_{\rho}(x)\right)} \int_{B_{\rho}(x) \cap \Omega_{2 R}\left(y_{0}\right)}|\nabla u|^{2} \mu(x) d x & \leq 2\left[\frac{1}{\mu\left(B_{\rho}(x)\right)} \int_{\Omega_{\rho}(x)}|\nabla u-\nabla v|^{2} \mu(x)+\frac{1}{\mu\left(B_{\rho}(x)\right)} \int_{\Omega_{\rho}(x)}|\nabla v|^{2} \mu(x) d x\right] \\
& \leq 2\left[\mathcal{M}_{\mu, \Omega_{3 r / 2}}\left(|\nabla u-\nabla v|^{2}\right)(x)+C_{*} \frac{\mu\left(\Omega_{\rho}(x)\right)}{\mu\left(B_{\rho}(x)\right)}\right] \\
& \leq 4 C_{*} \leq N_{1} .
\end{aligned}
$$

Now, if $\rho \geq r / 2$, then $B_{\rho}(x) \subset B_{5 \rho}\left(x_{0}\right)$. Therefore, it follows from 4.11) and Lemma2.2 that

$$
\begin{aligned}
\frac{1}{\mu\left(B_{\rho}(x)\right)} \int_{B_{\rho}(x) \cap \Omega_{2 R}\left(y_{0}\right)}|\nabla u|^{2} \mu(x) & \leq \frac{\mu\left(B_{5 \rho}\left(x_{0}\right)\right)}{\mu\left(B_{\rho}(x)\right)} \frac{1}{\mu\left(B_{5 \rho}\left(x_{0}\right)\right)} \int_{B_{5 \rho\left(x_{0}\right)} \cap \Omega_{2 R}\left(y_{0}\right)}|\nabla u|^{2} \mu(x) d x \\
& \leq \frac{\mu\left(B_{5 \rho}\left(x_{0}\right)\right)}{\mu\left(B_{\rho}(x)\right)} \mathcal{M}_{\mu, \Omega_{2 R}\left(y_{0}\right)}\left(\left.\nabla u\right|^{2}\right)\left(x_{0}\right) \leq 5^{2 n} M_{0} \leq N_{1},
\end{aligned}
$$

This estimates proves (4.14), and therefore implies (4.13). By taking the complement of both sets in (4.13) in $\Omega_{r}$, we obtain

$$
\left\{x \in \Omega_{r}: \mathcal{M}_{\mu, \Omega_{2 R}\left(y_{0}\right)}\left(|\nabla u|^{2}\right)<N_{1}\right\} \subset E:=\left\{x \in \Omega_{r}: \mathcal{M}_{\mu, \Omega_{3 r / 2}}\left(|\nabla u-\nabla v|^{2}\right)>C_{*}\right\} .
$$

On the other hand, by the weak type (1,1)-estimate, the doubling property in Lemma 2.2, (4.12), we also have

$$
\mu(E) \leq \frac{C\left(M_{0}\right) \mu\left(B_{3 r / 2}\right)}{C_{*}} \frac{1}{\mu\left(B_{3 r / 2}\right)} \int_{\Omega_{3 r / 2}}|\nabla u-\nabla v|^{2} \mu(x) d x \leq C^{\prime}\left(\Lambda, M_{0}, n\right) \gamma \mu\left(B_{r}\right) .
$$

Hence,

$$
\frac{\mu(E)}{\mu\left(B_{r}\right)} \leq C^{\prime}\left(\Lambda, M_{0}, n\right) \gamma
$$


From this, 4.15), and Lemma 2.3, there is $\beta=\beta\left(M_{2}, n\right)$ such that

$$
\begin{aligned}
\frac{\omega\left(\left\{x \in \Omega_{R}\left(y_{0}\right): \mathcal{M}_{\mu, \Omega_{2 R}\left(y_{0}\right)}\left(|\nabla u|^{2}\right)<N_{1}\right\} \cap B_{r}\right)}{\omega\left(B_{r}\right)} & \leq \frac{\omega\left(\left\{x \in \Omega_{r}: \mathcal{M}_{\mu, \Omega_{2 R}\left(y_{0}\right)}\left(|\nabla u|^{2}\right)<N_{1}\right\}\right.}{\omega\left(B_{r}\right)} \\
& \leq C\left(M_{0}, M_{2}\right)\left(\frac{\mu(E)}{\mu\left(B_{r}\right)}\right)^{\beta / 2} \\
& \leq C_{0}\left(\Lambda, M_{0}, M_{2}, n\right) \gamma^{\beta / 2} .
\end{aligned}
$$

Hence,

$$
\omega\left(\left\{x \in \Omega_{R}\left(y_{0}\right): \mathcal{M}_{\mu, \Omega_{2 R}\left(y_{0}\right)}\left(|\nabla u|^{2}\right)<N\right\} \cap B_{r}\right) \leq C_{0}\left(\Lambda, M_{0}, M_{2}, n\right) \gamma^{\beta / 2} .
$$

Therefore, by choosing $\gamma$ such that $C_{0}\left(\Lambda, M_{0}, M_{2}, n\right) \gamma^{\beta / 2}=\epsilon$, the lemma follows.

The following version of Lemma 3.5 is needed, and we state it for later reference.

Lemma 4.7. Let $\Lambda, M_{0} \geq 1, M_{1}>0, M_{2} \geq 1$ and sufficient $\epsilon>0$. There exist $N_{2}=N_{2}\left(\Lambda, M_{0}, n\right)>1$ and sufficiently small $\delta_{2}=\delta_{2}\left(\epsilon, \Lambda, M_{0}, M_{2}, n\right)>0$ such that the following statement holds: Let $\mathbb{K} \subset \mathbb{R}$ be some open interval, and let $\omega_{0}: \mathbb{K} \times[0, \infty) \rightarrow[0, \infty)$ be continuous satisfying (1.7) and $\left\|\omega_{0}\right\|_{\infty} \leq M_{1}$, there exists a large number $\lambda_{2}=\lambda_{2}\left(\epsilon, \Lambda, M_{0}, M_{1}, M_{2}, \omega_{0}, n\right) \geq 1$ such that for every $y_{0} \in \bar{\Omega}$ and $R>0$, if $\mathbb{A} \in \mathbb{U}_{\Omega_{2 R}\left(y_{0}\right), \mathbb{K}}\left(\Lambda, M_{0}, M_{1}, \omega_{0}\right)$ with its asymptotical matrix $\tilde{\mathbb{A}}$ and weight function $\mu \in A_{2}$ satisfying

$$
\sup _{0<\rho<R_{0}} \sup _{x \in \Omega_{R}\left(y_{0}\right)} \frac{1}{\mu\left(B_{\rho}(x)\right)} \int_{B_{\rho}(x) \cap \Omega}\left|\tilde{\mathbb{A}}(z)-\langle\tilde{\mathbb{A}}\rangle_{B_{\rho}(x) \cap \Omega}\right|^{2} \mu^{-1}(z) d z \leq \delta_{2}, \quad \text { for some } \quad R_{0}>0,
$$

and if $\lambda \geq \lambda_{2}$, and $u \in W^{1,2}\left(\Omega_{2 R}\left(y_{0}\right), \mu\right)$ a weak solution of (4.9) so that with some $y \in \Omega_{R}\left(y_{0}\right), 0<r<$ $\left.\min \left\{R_{0}, R\right\} / 3\right)$ such that $B_{3 r}(y) \subset \Omega_{2 R}\left(y_{0}\right)$, and

$$
B_{r}(y) \cap\left\{\Omega_{R}\left(y_{0}\right): \mathcal{M}_{\mu, \Omega_{2 R}\left(y_{0}\right)}\left(|\nabla u|^{2}\right) \leq 1\right\} \cap\left\{\Omega_{R}\left(y_{0}\right): \mathcal{M}_{\mu, \Omega_{2 R}\left(y_{0}\right)}\left(|\mathbf{F} / \mu|^{2}\right) \leq \delta_{2}\right\} \neq \emptyset,
$$

then

$$
\omega\left(\left\{x \in \Omega_{R}\left(y_{0}\right): \mathcal{M}_{\mu, \Omega_{2 R}\left(y_{0}\right)}\left(|\nabla u|^{2}\right)>N_{2}\right\} \cap B_{r}(y)\right)<\epsilon \omega\left(B_{\rho}(y)\right),
$$

for every weight function $\omega \in A_{q}$ with $[\omega]_{A_{q}} \leq M_{2}$, for $1 \leq q \leq \infty$.

Proof. The same as that of Lemma3.5, with $B_{R}$ replaced by $\Omega_{R}\left(y_{0}\right)$, and $B_{2 R}$ replaced by $\Omega_{2 R}\left(y_{0}\right)$.

Combining Lemma 4.6 and Lemma4.7, we can prove the following lemma

Lemma 4.8. Let $\Lambda>0, q \geq 1, M_{0} \geq 1, M_{2} \geq 1, M_{1}>0$ and let $\omega_{0}: \mathbb{K} \times[0, \infty) \rightarrow[0, \infty)$ be continuous, satisfy (1.7) and $\left\|\omega_{0}\right\|_{\infty} \leq M_{1}$ with some open interval $\mathbb{K} \subset \mathbb{R}$. Then, for every $\epsilon>0$, there are $N=$ $N\left(\Lambda, M_{0}, n\right), \delta=\delta\left(\epsilon, q, \Lambda, M_{0}, M_{2}, n\right) \in(0,1 / 8)$, and $\lambda_{0}=\lambda_{0}\left(\epsilon, \Lambda, M_{0}, M_{1}, M_{2}, \omega_{0}, q, n\right) \geq 1$ such that the following statement holds: Suppose $\Omega$ is $\left(\delta, R_{0}\right)$-Reifenberg flat in $\mathbb{R}^{n}$ for some $R_{0}>0$, and suppose that $\mathbb{A} \in \mathbb{U}_{\Omega, \mathbb{R}}\left(\Lambda, M_{0}, M_{1}, \omega_{0}\right)$ with its asymptotical matrix $\tilde{\mathbb{A}}$ and weight $\mu$ satisfying

$$
\sup _{0<\rho<R_{0}} \sup _{x \in \Omega} \frac{1}{\mu\left(B_{\rho}(x)\right)} \int_{\Omega_{\rho}(x)}\left|\tilde{\mathbb{A}}(y)-\langle\tilde{\mathbb{A}}\rangle_{\Omega_{\rho}(x)}\right|^{2} \mu^{-1}(y) d y \leq \delta .
$$

If $y_{0} \in \bar{\Omega}, R>0, \lambda \geq \lambda_{0}$, and $u \in W^{1,2}(\Omega, \mu)$ is a weak solution of (4.9) so that for some $y \in \Omega_{R}\left(y_{0}\right)$, $0<r<\min \left\{R_{0}, R\right\} / 50$,

$$
\omega\left(\left\{x \in \Omega_{R}\left(y_{0}\right): \mathcal{M}_{\mu, \Omega_{2 R}\left(y_{0}\right)}\left(|\nabla u|^{2}\right)(x)>N\right\} \cap B_{r}(y)\right) \geq \epsilon \omega\left(B_{r}(y)\right)
$$

for some $\omega \in A_{q}$ with $[\omega]_{A_{q}} \leq M_{2}$, then

$$
\begin{aligned}
\Omega_{r}(y) \subset & \left\{\Omega_{R}\left(y_{0}\right): \mathcal{M}_{\mu, \Omega_{2 R}\left(y_{0}\right)}\left(|\nabla u|^{2}\right)>1\right\} \cup \\
& \cup\left\{\Omega_{R}\left(y_{0}\right): \mathcal{M}_{\mu, \Omega_{2 R}\left(y_{0}\right)}\left(|\mathbf{F} / \mu|^{2}\right)>\delta\right\} \cup\left\{\Omega_{R}\left(y_{0}\right): \mathcal{M}_{\mu, \Omega_{2 R}\left(y_{0}\right)}\left(|\nabla g|^{2}\right)>\delta^{2}\right\} .
\end{aligned}
$$


Proof. Let $N=\max \left\{N_{1}, N_{2}\right\}, \epsilon^{\prime}=\frac{\epsilon}{M_{2}(17)^{q n}}$, and

$$
\begin{aligned}
& \delta=\min \left\{\delta_{1}\left(\epsilon^{\prime}, \Lambda, M_{0}, M_{2}, n\right), \delta_{2}\left(\epsilon, \Lambda, M_{0}, M_{2}, n\right), 1 / 8\right\}, \quad \text { and } \\
& \lambda_{0}=\max \left\{\lambda_{1}\left(\epsilon^{\prime}, \Lambda, M_{0}, M_{1}, M_{2}, \omega_{0}, n\right), \lambda_{2}\left(\epsilon, \Lambda, M_{0}, M_{1}, M_{2}, \omega_{0}, n\right)\right\},
\end{aligned}
$$

where $N_{1}, \delta_{1}, \lambda_{1}$ are defined in Lemma4.6 and $N_{2}, \delta_{2}, \lambda_{2}$ are defined in Lemma4.7 We observe that since $\epsilon^{\prime}$ depends on $q$, so do $\delta, \lambda_{0}$.

Since $y \in \Omega_{R}\left(y_{0}\right)$, and $0<r<\min \left\{R_{0}, R\right\} / 50$, we see that $B_{3 r}(y) \subset B_{2 R}\left(y_{0}\right)$. Therefore, if $B_{3 r}(y) \cap \partial \Omega=\emptyset$, Lemma 4.8 follows directly by Lemma 4.7 and our choice of $N, \delta, \lambda_{0}$. It then remains to consider the case that $B_{3 r}(y) \cap \partial \Omega \neq \emptyset$. In this case, we complete the proof by a contradiction argument. Assume there is $x_{0} \in \Omega_{r}(y)$ so that

$$
\mathcal{M}_{\mu, \Omega_{2 R}\left(y_{0}\right)}\left(|\nabla u|^{2}\right)\left(x_{0}\right) \leq 1, \quad \mathcal{M}_{\mu, \Omega_{2 R}\left(y_{0}\right)}\left(|\mathbf{F} / \mu|^{2}\right)\left(x_{0}\right) \leq \delta, \quad \text { and } \quad \mathcal{M}_{\mu, \Omega_{2 R}\left(y_{0}\right)}\left(|\nabla g|^{2}\right)\left(x_{0}\right) \leq \delta .
$$

Because $B_{3 r}(y) \cap \partial \Omega \neq \emptyset$, we can find $\tilde{y}_{0} \in \partial \Omega \cap B_{3 r}(y)$. We observe that

$$
x_{0} \in \Omega_{r}(y)=B_{r}(y) \cap \Omega \subset B_{4 r}\left(\tilde{y}_{0}\right) \cap \Omega=\Omega_{4 r}\left(\tilde{y}_{0}\right) .
$$

Let $\rho=7 r$, we observe that $3 \rho<R_{0} / 2$. Since $\Omega$ is $\left(\delta, R_{0}\right)$ Reifenberg flat domain, and by Remark 2.9 , there exists an orthonormal coordinate system $\left\{\vec{z}_{1}, \vec{z}_{2}, \cdots, \vec{z}_{n}\right\}$ in which $0 \in \Omega$,

$$
\tilde{y}_{0}=-3 \rho \delta \vec{z}_{n} \in \partial \Omega, \quad y_{0}=z, \quad y=\hat{z}, \quad x_{0}=z_{0},
$$

and

In this new coordinate, the assumption (4.18) becomes

$$
B_{3 \rho}^{+}(0) \subset \Omega_{3 \rho} \subset B_{3 \rho} \cap\left\{z_{n}>-12 \rho \delta\right\} .
$$

$$
\omega\left(\left\{\Omega_{R}(z): \mathcal{M}_{\mu, \Omega_{2 R}(z)}\left(|\nabla u|^{2}\right)>N\right\} \cap B_{r}(\hat{z})\right) \geq \epsilon \omega\left(B_{r}(\hat{z})\right) .
$$

We also observe that in the new coordinate system $z_{0} \in B_{\rho}(0)$. Indeed,

$$
|\hat{z}|<\left|\hat{z}-\tilde{y}_{0}\right|+\left|\tilde{y}_{0}\right|=\left|y-\tilde{y}_{0}\right|+\left|\tilde{y}_{0}\right| \leq 3 r+3 \rho \delta,
$$

and therefore

$$
\left|z_{0}\right| \leq\left|z_{0}-\hat{z}\right|+|\hat{z}| \leq 4 r+3 \rho \delta \leq 4 r+\frac{3}{8} \rho<\rho .
$$

Collecting all estimates, after a change of coordinate system, and after a simple calculation, we obtain the followings

(i) $u \in W^{1,2}(\Omega, \mu)$ is a weak solution to [4.9), $0 \in \Omega$,

(ii) $B_{3 \rho}^{+}(0) \subset \Omega_{3 \rho} \subset B_{3 \rho}(0) \cap\left\{z=\left(z^{\prime}, z_{n}\right): z_{n}>-12 \rho \delta\right\}$,

(iii) $z_{0} \in B_{\rho}(0) \cap\left\{\Omega_{R}(z): \mathcal{M}_{\mu, \Omega_{2 R}(z)}\left(|\nabla u|^{2}\right) \leq 1\right\}$ and

$z_{0} \in\left\{\Omega_{R}(z): \mathcal{M}_{\mu, \Omega_{2 R}(z)}\left(|\mathbf{F} / \mu|^{2}\right) \leq \delta\right\} \cap\left\{\Omega_{R}(z): \mathcal{M}_{\mu, \Omega_{2 R}(z)}\left(|\nabla g|^{2}\right) \leq \delta\right\}$, and

(iv) $B_{r}(\hat{z}) \subset B_{\rho}(0) \subset B_{17 r}(\hat{z})$.

From (i)-(iii), and the choice of $\delta$, we see that all the hypotheses of Lemma 4.6 are satisfied. We thus conclude that

$$
\omega\left(B_{\rho}(0) \cap\left\{\Omega_{R}(z): \mathcal{M}_{\mu, \Omega_{2 R}(z)}\left(|\nabla u|^{2}\right)>N\right\}\right)<\epsilon^{\prime} \omega\left(B_{\rho}(0)\right) .
$$

Moreover, from item (iv), we infer that

$$
\begin{aligned}
\omega\left(B_{r}(\hat{z}) \cap\left\{\Omega_{R}(z): \mathcal{M}_{\mu, \Omega_{2 R}(z)}\left(|\nabla u|^{2}\right)>N\right\}\right) & \left.\leq \omega\left(B_{\rho}(0) \cap\left\{\Omega_{R}(z): M_{\mu, \Omega_{2 R}(z)}|\nabla u|^{2}\right)>N\right\}\right) \\
& <\frac{\epsilon}{M_{2} 17^{q n}} \omega\left(B_{\rho}(0)\right) \leq \frac{\epsilon}{M_{2} 17^{q n}} \omega\left(B_{17 r}(\hat{z})\right) \\
& \leq \frac{\epsilon}{M_{2} 17^{q n}} M_{2} 17^{q n} \mu\left(B_{r}(\hat{z})\right) \\
& =\epsilon \mu\left(B_{r}(\hat{z})\right),
\end{aligned}
$$

where we have used the doubling property of the $\mu$ as in Lemma2.2. The last estimate obviously contradicts (4.19), and thus the proof is complete. 
Finally, we can estimate the density of the level sets of $\mathcal{M}_{\mu, \Omega_{2 R}\left(y_{0}\right)}\left(|\nabla u|^{2}\right)$, which is the main result of the subsection.

Proposition 4.9. Let $A>0, q \geq 1, \Lambda>0, M_{0} \geq 1, M_{2} \geq 1, M_{1}>0$, and let $\epsilon>0$ sufficiently small. Also, let $\omega_{0}: \mathbb{K} \times[0, \infty) \rightarrow[0, \infty)$ be continuous, satisfy $\left\|\omega_{0}\right\|_{\infty} \leq M_{1}$ with some open interval $\mathbb{K} \subset \mathbb{R}$, and then let $N=N\left(\Lambda, M_{0}, n\right) \geq 1, \delta=\delta\left(\epsilon, q, \Lambda, M_{0}, M_{2}, n\right) \in(0,1 / 8)$, and $\lambda_{0}=\lambda_{0}\left(\epsilon, \Lambda, M_{0}, M_{1}, M_{2}, \omega_{0}, q, n\right) \geq 1$ be as in Lemma 4.8. Suppose that $\Omega$ is $\left(\delta, R_{0}\right)$-Reifenberg flat in $\mathbb{R}^{n}$ for some $R_{0}>0$, and $\mathbb{A} \in \mathbb{U}_{\Omega, \mathbb{K}}\left(\Lambda, M_{0}, M_{1}, \omega_{0}\right)$ with its asymptotical matrix $\tilde{\mathbb{A}}$ and weight $\mu$ satisfying

$$
\sup _{0<\rho<R_{0}} \sup _{x \in \Omega} \frac{1}{\mu\left(B_{\rho}(x)\right)} \int_{\Omega_{\rho}(x)}\left|\tilde{\mathbb{A}}(y)-\langle\tilde{\mathbb{A}}\rangle_{\Omega_{\rho}(x)}\right|^{2} \mu^{-1}(y) d y \leq \delta .
$$

Then, for any $\lambda \geq \lambda_{0}$, if $u \in W^{1,2}(\Omega, \mu)$ is a weak solution of (4.9) such that for some $R>0, y_{0} \in \bar{\Omega}$, some fixed $0<r_{0}<\min \left\{R_{0}, R\right\} / 50$, and some $\omega \in A_{q}$ satisfying $[\omega]_{A_{q}} \leq M_{2}$,

$$
\omega\left(\left\{\Omega_{R}\left(y_{0}\right): \mathcal{M}_{\mu, \Omega_{2 R}\left(y_{0}\right)}\left(|\nabla u|^{2}\right)>N\right\}\right) \leq \epsilon \omega\left(B_{r_{0}}(y)\right), \quad \forall y \in \bar{\Omega}_{R}\left(y_{0}\right),
$$

and $\Omega_{R}\left(y_{0}\right)$ is of $\left(A, r_{0}\right)$ type, then with $\epsilon_{1}=5^{n q} \max \left\{A^{-1}, 4^{n}\right\}^{q} M_{2}^{2} \epsilon$,

$$
\begin{aligned}
& \omega\left(\left\{\Omega_{R}\left(y_{0}\right): \mathcal{M}_{\mu, \Omega_{2 R}\left(y_{0}\right)}\left(|\nabla u|^{2}>N\right\}\right)\right. \\
& \leq \epsilon_{1}\left[\omega\left(\left\{\Omega_{R}\left(y_{0}\right): \mathcal{M}_{\mu, \Omega_{2 R}\left(y_{0}\right)}\left(|\nabla u|^{2}\right)>1\right\}\right)\right. \\
& \left.\quad+\omega\left(\left\{\Omega_{R}\left(y_{0}\right): \mathcal{M}_{\mu, \Omega_{2 R}\left(y_{0}\right)}\left(|\nabla g|^{2}\right)>\delta\right\}\right)+\omega\left(\left\{\Omega_{R}\left(y_{0}\right): \mathcal{M}_{\mu, \Omega_{2 R}\left(y_{0}\right)}\left(|\mathbf{F} / \mu|^{2}\right)>\delta\right\}\right)\right] .
\end{aligned}
$$

Proof. Let us also denote

$$
C=\left\{\Omega_{R}\left(y_{0}\right): \mathcal{M}_{\mu, \Omega_{2 R}\left(y_{0}\right)}\left(|\nabla u|^{2}\right)>N\right\},
$$

and

$$
\begin{aligned}
D=\{ & \left.\Omega_{R}\left(y_{0}\right): \mathcal{M}_{\mu, \Omega_{2 R}\left(y_{0}\right)}\left(|\nabla u|^{2}\right)>1\right\} \cup \\
& \cup\left\{\Omega_{R}\left(y_{0}\right): \mathcal{M}_{\mu, \Omega_{2 R}\left(y_{0}\right)}\left(|\nabla g|^{2}\right)>\delta\right\} \cup\left\{\Omega_{R}\left(y_{0}\right): \mathcal{M}_{\mu, \Omega_{2 R}\left(y_{0}\right)}\left(|\mathbf{F} / \mu|^{2}\right)>\delta\right\} .
\end{aligned}
$$

Clearly $C \subset D \subset \Omega_{R}\left(y_{0}\right)$. Moreover, by the assumption, we see that $\omega(C)<\epsilon \omega\left(B_{r_{0}}(y)\right)$ for all $y \in \bar{\Omega}_{R}\left(y_{0}\right)$. Therefore, (i) of Lemma 2.7 holds. Moreover, it is simple to check that (ii) follows from Lemma 4.8 . Therefore, Lemma 4.9 is just a consequence of Lemma 2.7

4.3. Proof of the global weighted $W^{1, p}$-regularity estimates. From the Proposition 4.9 and an iterating procedure, we obtain the following lemma

Lemma 4.10. Let $A, \Lambda>0, M_{0}, M_{2}, q \geq 1, M_{1}>0$, and let $\epsilon>0$ sufficiently small. Also, let $\omega_{0}: \mathbb{K} \times$ $[0, \infty) \rightarrow[0, \infty)$ be continuous, satisfy $\left\|\omega_{0}\right\|_{\infty} \leq M_{1}$ with some open interval $\mathbb{K} \subset \mathbb{R}$, and let $N=N\left(\Lambda, M_{0}, n\right)$, $\delta=\delta\left(\epsilon, q, \Lambda, M_{0}, M_{2}, n\right) \in(0,1 / 8)$, and $\lambda_{0}=\lambda_{0}\left(\epsilon, \Lambda, M_{0}, M_{1}, M_{2}, \omega_{0}, q, n\right)$ be as in Proposition 4.9 Suppose that $\Omega$ is $\left(\delta, R_{0}\right)$-Reifenberg flat in $\mathbb{R}^{n}$ for some $R_{0}>0$, and $\mathbb{A} \in \mathbb{U}_{\Omega, \mathbb{K}}\left(\Lambda, M_{0}, M_{1}, \omega_{0}\right)$ with its asymptotical matrix $\tilde{\mathbb{A}}$ and weight $\mu$ satisfying

$$
\sup _{0<\rho<R_{0}} \sup _{x \in \Omega} \frac{1}{\mu\left(B_{\rho}(x)\right)} \int_{\Omega_{\rho}(x)}\left|\tilde{\mathbb{A}}(y)-\langle\tilde{\mathbb{A}}\rangle_{B_{\rho}(x)}\right|^{2} \mu^{-1}(y) d y \leq \delta .
$$

Then, for any $\lambda \geq \lambda_{0}, y \in \bar{\Omega}$ and $R>0$ such that $\Omega_{R}\left(y_{0}\right)$ is of type $\left(A, r_{0}\right)$ for some fixed $0<r_{0}<$ $\min \left\{R_{0}, R\right\} / 50$, if $u \in W^{1,2}(\Omega, \mu)$ is a weak solution of (4.9) such that for some $\omega \in A_{q}$ satisfying $[\omega]_{A_{q}} \leq M_{2}$,

$$
\omega\left(\left\{x \in \mathbb{R}^{n}: \mathcal{M}_{\mu, \Omega_{2 R}\left(y_{0}\right)}\left(|\nabla u|^{2}\right)>N\right\}\right) \leq \epsilon \omega\left(B_{r_{0}}(y)\right), \quad \forall y \in \bar{\Omega}_{R}\left(y_{0}\right),
$$


then with $\epsilon_{1}=5^{n q} \max \left\{A^{-1}, 4^{n}\right\}^{q} M_{2}^{2} \epsilon$, and for any $k \in \mathbb{N}$,

$$
\begin{aligned}
& \omega\left(\left\{\Omega_{R}\left(y_{0}\right): \mathcal{M}_{\mu, \Omega_{2 R}\left(y_{0}\right)}\left(|\nabla u|^{2}>N^{k}\right\}\right) \leq \epsilon_{1}^{k} \omega\left(\left\{\Omega_{R}\left(y_{0}\right): \mathcal{M}_{\mu, \Omega_{2 R}\left(y_{0}\right)}\left(|\nabla u|^{2}\right)>1\right\}\right)\right. \\
& \quad+\sum_{i=1}^{k} \epsilon_{1}^{i}\left[\omega\left(\left\{\Omega_{R}\left(y_{0}\right): \mathcal{M}_{\mu, \Omega_{2 R}\left(y_{0}\right)}\left(|\nabla g|^{2}\right)>\delta N^{k-i}\right\}\right)+\omega\left(\left\{\Omega_{R}\left(y_{0}\right): \mathcal{M}_{\mu, \Omega_{2 R}\left(y_{0}\right)}\left(|\mathbf{F} / \mu|^{2}\right)>\delta N^{k-i}\right\}\right)\right] .
\end{aligned}
$$

Proof. We use induction on $k$. If $k=1$, 4.22 holds as a result of Proposition 4.9. Now, let us assume that Lemma 4.10 holds for some $k \in\left\{1,2, \cdots, k_{0}\right\}$ with some $k_{0} \in \mathbb{N}$. Assume that $u$ is a weak solution of (4.9) with some $\lambda \geq \lambda_{0}$ so that (4.21) holds. Now, let us define $u^{\prime}=u / \sqrt{N}, \mathbf{F}^{\prime}=\mathbf{F} / \sqrt{N}, g^{\prime}=g / \sqrt{N}$, and $\lambda^{\prime}=\lambda \sqrt{N} \geq \lambda \geq \lambda_{0}$. Then, we see that $u^{\prime}$ is a weak solution of

$$
\left\{\begin{array}{cccc}
\operatorname{div}\left[\mathbb{A}_{\lambda^{\prime}}\left(x, u^{\prime}, \nabla u^{\prime}\right)\right] & = & \operatorname{div}\left[\mathbf{F}^{\prime}\right], & \text { in } \Omega, \\
u^{\prime} & = & g^{\prime}, & \text { on } \partial \Omega .
\end{array}\right.
$$

Moreover, $\forall y \in \bar{\Omega}_{R}\left(y_{0}\right)$

$$
\omega\left(\left\{\Omega_{R}\left(y_{0}\right): \mathcal{M}_{\mu, \Omega_{2 R}\left(y_{0}\right)}\left(\left|\nabla u^{\prime}\right|^{2}\right)>N\right\}\right)=\omega\left(\left\{\Omega_{R}\left(y_{0}\right): \mathcal{M}_{\mu, \Omega_{2 R}\left(y_{0}\right)}\left(|\nabla u|^{2}\right)>N^{2}\right\}\right) \leq \epsilon \omega\left(B_{r_{0}}(y)\right) .
$$

Then, by applying the the induction hypothesis on $u^{\prime}$, we obtain

$$
\begin{aligned}
& \omega\left(\left\{\Omega_{R}\left(y_{0}\right): \mathcal{M}_{\mu, \Omega_{2 R}\left(y_{0}\right)}\left(\left|\nabla u^{\prime}\right|^{2}>N^{k_{0}}\right\}\right)\right. \\
& \leq \epsilon_{1}^{k_{0}} \omega\left(\left\{\Omega_{R}\left(y_{0}\right): \mathcal{M}_{\mu, \Omega_{2 R}\left(y_{0}\right)}\left(\left|\nabla u^{\prime}\right|^{2}\right)>1\right\}\right) \\
& +\sum_{i=1}^{k_{0}} \epsilon_{1}^{i}\left[\omega\left(\left\{\Omega_{R}\left(y_{0}\right): \mathcal{M}_{\mu, \Omega_{2 R}\left(y_{0}\right)}\left(\left|\nabla g^{\prime}\right|^{2}\right)>\delta N^{k_{0}-i}\right\}\right)+\omega\left(\left\{\Omega_{R}\left(y_{0}\right): \mathcal{M}_{\mu, \Omega_{2 R}\left(y_{0}\right)}\left(\left|\mathbf{F}^{\prime} / \mu\right|^{2}\right)>\delta N^{k_{0}-i}\right\}\right)\right] .
\end{aligned}
$$

This implies

$$
\begin{aligned}
& \omega\left(\left\{\Omega_{R}\left(y_{0}\right): \mathcal{M}_{\mu, \Omega_{2 R}\left(y_{0}\right)}\left(|\nabla u|^{2}>N^{k_{0}+1}\right\}\right)\right. \\
& \leq \epsilon_{1}^{k_{0}} \omega\left(\left\{\Omega_{R}\left(y_{0}\right): \mathcal{M}_{\mu, \Omega_{2 R}\left(y_{0}\right)}\left(|\nabla u|^{2}\right)>N\right\}\right) \\
& +\sum_{i=1}^{k_{0}} \epsilon_{1}^{i}\left[\omega\left(\left\{\Omega_{R}\left(y_{0}\right): \mathcal{M}_{\mu, \Omega_{2 R}\left(y_{0}\right)}\left(|\nabla g|^{2}\right)>\delta N^{k_{0}+1-i}\right\}\right)+\omega\left(\left\{\Omega_{R}\left(y_{0}\right): \mathcal{M}_{\mu, \Omega_{2 R}\left(y_{0}\right)}\left(\left|\mathbf{F}^{\prime} / \mu\right|^{2}\right)>\delta N^{k_{0}+1-i}\right\}\right)\right] .
\end{aligned}
$$

On the other hand, we also have

$$
\begin{aligned}
& \omega\left(\left\{\Omega_{R}\left(y_{0}\right): \mathcal{M}_{\mu, \Omega_{2 R}\left(y_{0}\right)}\left(|\nabla u|^{2}>N\right\}\right)\right. \\
& \leq \epsilon_{1}\left[\omega\left(\left\{\Omega_{R}\left(y_{0}\right): \mathcal{M}_{\mu, \Omega_{2 R}\left(y_{0}\right)}\left(|\nabla u|^{2}\right)>1\right\}\right)\right. \\
& \left.\quad+\omega\left(\left\{\Omega_{R}\left(y_{0}\right): \mathcal{M}_{\mu, \Omega_{2 R}\left(y_{0}\right)}\left(|\nabla g|^{2}\right)>\delta\right\}\right)+\omega\left(\left\{\Omega_{R}\left(y_{0}\right): \mathcal{M}_{\mu, \Omega_{2 R}\left(y_{0}\right)}\left(|\mathbf{F} / \mu|^{2}\right)>\delta\right\}\right)\right] .
\end{aligned}
$$

Therefore, by combining the last two estimates, we see that

$$
\begin{aligned}
& \omega\left(\left\{\Omega_{R}\left(y_{0}\right): \mathcal{M}_{\mu, \Omega_{2 R}\left(y_{0}\right)}\left(|\nabla u|^{2}>N^{k_{0}+1}\right\}\right)\right. \\
& \leq \epsilon_{1}^{k_{0}+1} \omega\left(\left\{\Omega_{R}\left(y_{0}\right): \mathcal{M}_{\mu, \Omega_{2 R}\left(y_{0}\right)}\left(|\nabla u|^{2}\right)>N\right\}\right) \\
& +\sum_{i=1}^{k_{0}+1} \epsilon_{1}^{i}\left[\omega\left(\left\{\Omega_{R}\left(y_{0}\right): \mathcal{M}_{\mu, \Omega_{2 R}\left(y_{0}\right)}\left(|\nabla g|^{2}\right)>\delta N^{k_{0}+1-i}\right\}\right)+\omega\left(\left\{\Omega_{R}\left(y_{0}\right): \mathcal{M}_{\mu, \Omega_{2 R}\left(y_{0}\right)}\left(\left|\mathbf{F}^{\prime} / \mu\right|^{2}\right)>\delta N^{k_{0}+1-i}\right\}\right)\right] .
\end{aligned}
$$

Hence, we have proved Lemma 4.10 for $k \leq k_{0}+1$. Lemma4.10 then holds for all $k \in \mathbb{N}$ by induction, and the proof is complete. 
Proof of Theorem 1.6. Let $N=N\left(\Lambda, M_{0}, n\right)$ be defined as in Lemma4.10. For $p>2$, we denote $s=p / 2>$ 1 , and choose $\epsilon>0$ and sufficiently small and depending only on $\Lambda, A, M_{0}, n, p, q$ such that

$$
\epsilon_{1} N^{s}=1 / 2,
$$

where $\epsilon_{1}$ is defined in Lemma4.10. With this $\epsilon$, we can now choose

$$
\delta=\delta\left(q, \Lambda, A, M_{0}, M_{2}, p, n\right), \quad \lambda_{0}=\lambda_{0}\left(\Lambda, A, M_{0}, M_{1}, M_{2}, \omega_{0}, p, q, n\right)
$$

as determined by Lemma4.10. For $\lambda \geq \lambda_{0}$, let us assume for a moment that $u$ is a weak solution of (4.9). Let

$$
E=E(\lambda, N)=\left\{\Omega_{R}\left(y_{0}\right): \mathcal{M}_{\mu, \Omega_{2 R}\left(y_{0}\right)}\left(|\nabla u|^{2}\right)>N\right\} .
$$

We can assume without loss of generality that $r_{0}<\min \left\{R, R_{0}\right\} / 50$. We first prove the estimate in Theorem 1.6 with the extra condition that

$$
\omega(E) \leq \epsilon \omega\left(B_{r_{0}}(y)\right), \quad \forall y \in \bar{\Omega}_{R}\left(y_{0}\right) .
$$

Let us now consider the sum

$$
S=\sum_{k=1}^{\infty} N^{s k} \omega\left(\left\{\Omega_{R}\left(y_{0}\right): \mathcal{M}_{\mu, \Omega_{2 R}\left(y_{0}\right)}\left(|\nabla u|^{2}\right)>N^{k}\right\}\right) .
$$

From 4.24), we can apply Lemma 4.10 to obtain

$$
\begin{aligned}
S \leq & \sum_{k=1}^{\infty} N^{k s}\left[\sum_{i=1}^{k} \epsilon_{1}^{i} \omega\left(\left\{\Omega_{R}\left(y_{0}\right): \mathcal{M}_{\mu, \Omega_{2 R}\left(y_{0}\right)}\left(|\mathbf{F} / \mu|^{2}\right)>\delta N^{k-i}\right\}\right)\right. \\
& \left.+\sum_{i=1}^{k} \epsilon_{1}^{i} \omega\left(\left\{\Omega_{R}\left(y_{0}\right): \mathcal{M}_{\mu, \Omega_{2 R}\left(y_{0}\right)}\left(|\nabla g|^{2}\right)>\delta N^{k-i}\right\}\right)\right] \\
& +\sum_{k=1}^{\infty}\left(N^{s} \epsilon_{1}\right)^{k} \omega\left(\left\{\Omega_{R}\left(y_{0}\right): \mathcal{M}_{\mu, \Omega_{2 R}\left(y_{0}\right)}\left(|\nabla u|^{2}\right)>1\right\}\right) .
\end{aligned}
$$

By Fubini's theorem, the above estimate can be rewritten as

$$
\begin{aligned}
S \leq & \sum_{j=1}^{\infty}\left(N^{s} \epsilon_{1}\right)^{j}\left[\sum_{k=j}^{\infty} N^{s(k-j)} \omega\left(\left\{\Omega_{R}\left(y_{0}\right): \mathcal{M}_{\mu, \Omega_{2 R}\left(y_{0}\right)}\left(|\mathbf{F} / \mu|^{2}\right)>\delta N^{k-j}\right\}\right)\right. \\
& \left.+\sum_{k=j}^{\infty} N^{s(k-j)} \omega\left(\left\{\Omega_{R}\left(y_{0}\right): \mathcal{M}_{\mu, \Omega_{2 R}}\left(|\nabla g|^{2}\right)>\delta N^{k-j}\right\}\right)\right] \\
& +\sum_{k=1}^{\infty}\left(N^{s} \epsilon_{1}\right)^{k} \omega\left(\left\{\Omega_{R}\left(y_{0}\right): \mathcal{M}_{\mu, \Omega_{2 R}\left(y_{0}\right)}\left(|\nabla u|^{2}\right)>1\right\}\right) .
\end{aligned}
$$

Observe that

$$
\omega\left(\left\{\Omega_{R}\left(y_{0}\right): \mathcal{M}_{\mu, \Omega_{2 R}\left(y_{0}\right)}\left(|\nabla u|^{2}\right)>1\right\}\right) \leq \omega\left(\Omega_{R}\left(y_{0}\right)\right) .
$$

From this, the choice of $\epsilon$, and Lemma 2.4, and 4.25) it follows that

$$
S \leq C\left[\left\|\mathcal{M}_{\mu, \Omega_{2 R}\left(y_{0}\right)}\left(|\mathbf{F} / \mu|^{2}\right)\right\|_{L^{s}\left(\Omega_{R}\left(y_{0}\right), \omega\right)}^{s}+\left\|\mathcal{M}_{\mu, \Omega_{2 R}\left(y_{0}\right)}\left(|\nabla g|^{2}\right)\right\|_{L^{s}\left(\Omega_{R}\left(y_{0}\right), \omega\right)}^{s}+\omega\left(\Omega_{R}\left(y_{0}\right)\right)\right] .
$$

Applying the Lemma2.4 again, we see that

$$
\left\|\mathcal{M}_{\mu, \Omega_{2 R}\left(y_{0}\right)}\left(|\nabla u|^{2}\right)\right\|_{L^{s}\left(\Omega_{R}\left(y_{0}\right), \omega\right)}^{s} \leq C\left[\left\|\mathcal{M}_{\mu, \Omega_{2 R}\left(y_{0}\right)}\left(|\mathbf{F} / \mu|^{2}\right)\right\|_{L^{s}\left(\Omega_{2 R}\left(y_{0}\right), \omega\right)}^{s}+\left\|\mathcal{M}_{\mu, \Omega_{2 R}\left(y_{0}\right)}\left(|\nabla g|^{2}\right)\right\|_{L^{s}\left(\Omega_{2 R}\left(y_{0}\right), \omega\right)}^{s}+\omega\left(\Omega_{R}\left(y_{0}\right)\right)\right] \text {. }
$$

By the Lesbegue's differentiation theorem, we observe that

$$
|\nabla u(x)|^{2} \leq \mathcal{M}_{\mu, \Omega_{2 R}\left(y_{0}\right)}\left(|\nabla u|^{2}\right)(x), \quad \text { a.e } x \in \Omega_{R}\left(y_{0}\right) .
$$


Hence,

$$
\|\nabla u\|_{L^{p}\left(\Omega_{R}\left(y_{0}\right), \omega\right)}^{p} \leq C\left[\left\|\mathcal{M}_{\mu, \Omega_{2 R}\left(y_{0}\right)}\left(|\mathbf{F} / \mu|^{2}\right)\right\|_{L^{s}\left(\Omega_{R}\left(y_{0}\right), \omega\right)}^{s}+\left\|\mathcal{M}_{\mu, \Omega_{2 R}\left(y_{0}\right)}\left(|\nabla g|^{2}\right)\right\|_{L^{s}\left(\Omega_{R}\left(y_{0}\right), \omega\right)}^{s}+\omega\left(\Omega_{R}\left(y_{0}\right)\right)\right] .
$$

Since $(\mu, \omega)$ satisfies the $s$-Sawyer's condition, by Theorem 2.6 it follows

$$
\|\nabla u\|_{L^{p}\left(\Omega_{R}\left(y_{0}\right), \omega\right)} \leq C\left[\|\mathbf{F} / \mu\|_{L^{p}\left(\Omega_{2 R}\left(y_{0}\right), \omega\right)}+\|\nabla g\|_{L^{p}\left(\Omega_{2 R}\left(y_{0}\right), \omega\right)}+\omega\left(\Omega_{R}\left(y_{0}\right)\right)^{1 / p}\right] .
$$

Thus, we have proved (4.26) as long as $u$ is a weak solution of (4.9) for all $\lambda \geq \lambda_{0}$ and (4.24) holds.

Next, we consider the case $0<\lambda<\lambda_{0}$. Assume that $u$ is a weak solution of (4.9) and (4.24) holds. Let us denote $u^{\prime}=u /\left(\lambda_{0} / \lambda\right), \mathbf{F}^{\prime}=\mathbf{F} /\left(\lambda_{0} / \lambda\right), g^{\prime}=g /\left(\lambda_{0} / \lambda\right)$. Then, $u^{\prime}$ is a weak solution of

$$
\left\{\begin{array}{cccc}
\operatorname{div}\left[\mathbb{A}_{\lambda_{0}}\left(x, u^{\prime}, \nabla u^{\prime}\right)\right] & = & \operatorname{div}\left[\mathbf{F}^{\prime}\right], & \text { in } \Omega, \\
u^{\prime} & = & g^{\prime}, & \text { on } \Omega .
\end{array}\right.
$$

Moreover, because of (4.24) and $\lambda_{0} / \lambda \geq 1$, we also have

$$
\omega\left(\Omega_{R}\left(y_{0}\right):\left\{\mathcal{M}_{\mu, \Omega_{2 R}\left(y_{0}\right)}\left(\left|\nabla u^{\prime}\right|^{2}\right)>N\right\}\right) \leq \epsilon \omega\left(B_{r_{0}}(y)\right), \quad \forall y \in \bar{\Omega}_{R}\left(y_{0}\right) .
$$

Therefore, applying the conclusion of (4.26) for $u^{\prime}$, we also obtain

$$
\left\|\nabla u^{\prime}\right\|_{L^{p}\left(\Omega_{R}\left(y_{0}\right), \omega\right)} \leq C\left[\left\|\mathbf{F}^{\prime} / \mu\right\|_{L^{p}\left(\Omega_{2 R}\left(y_{0}\right), \omega\right)}+\left\|\nabla g^{\prime}\right\|_{L^{p}\left(\Omega_{2 R}\left(y_{0}\right), \omega\right)}+\omega\left(\Omega_{R}\left(y_{0}\right)\right)^{1 / p}\right] .
$$

Thus,

$$
\|\nabla u\|_{L^{p}\left(\Omega_{R}\left(y_{0}\right), \omega\right)} \leq C\left[\|\mathbf{F} / \mu\|_{L^{p}\left(\Omega_{2 R}\left(y_{0}\right), \omega\right)}+\|\nabla g\|_{L^{p}\left(\Omega_{2 R}\left(y_{0}\right), \omega\right)}+\lambda_{0} \omega\left(\Omega_{R}\left(y_{0}\right)\right)^{1 / p} / \lambda\right] .
$$

In summary, up to now, we have proved that if $u$ is a weak solution of (4.9) with $\lambda>0$ and if (4.24) holds, then

$$
\|\nabla u\|_{L^{p}\left(\Omega_{R}\left(y_{0}\right), \omega\right)} \leq C\left[\|\mathbf{F} / \mu\|_{L^{p}\left(\Omega_{2 R}\left(y_{0}\right), \omega\right)}+\|\nabla g\|_{L^{p}\left(\Omega_{2 R}\left(y_{0}\right), \omega\right)}+\omega\left(\Omega_{R}\left(y_{0}\right)\right)^{1 / p} \max \left\{\lambda^{-1}, 1\right\}\right] .
$$

We now remove the extra condition (4.24). Assuming now that $u$ is a weak solution of (1.1), i.e. $\lambda=1$. Let $M>1$ sufficiently large and will be determined. Let $u_{M}=u / M, \mathbf{F}_{M}=\mathbf{F} / M$ and $g_{M}=g / M$. We note that $u_{M}$ is a weak solution of

$$
\left\{\begin{array}{cccc}
\operatorname{div}\left[\mathbb{A}_{M}\left(x, u_{M}, \nabla u_{M}\right)\right] & = & \operatorname{div}\left[\mathbf{F}_{M}\right], & \text { in } \Omega, \\
u_{M} & = & g_{M}, & \text { on } \partial \Omega .
\end{array}\right.
$$

Let us denote

$$
E_{M}=\left\{\Omega_{R}\left(y_{0}\right): \mathcal{M}_{\mu, \Omega_{2 R}\left(y_{0}\right)}\left(\left|\nabla u_{M}\right|^{2}\right)>N\right\}
$$

and

$$
K_{0}=\left(\frac{1}{\mu\left(B_{2 R}\left(y_{0}\right)\right)} \int_{\Omega_{2 R}\left(y_{0}\right)}|\nabla u|^{2} \mu(x) d x\right)^{1 / 2}+1 \geq 1 .
$$

We claim that we can choose $M=C K_{0}$ with some sufficiently large constant $C$ depending only on $\Lambda, M_{0}, M_{1}$, $M_{2}, p, q, n$ and $R / r_{0}$ such that

$$
\omega\left(E_{M}\right) \leq \epsilon \omega\left(B_{r_{0}}(y)\right), \quad \forall y \in \bar{\Omega}_{R}\left(y_{0}\right) .
$$

If this holds, we can apply (4.27) for $u_{M}$ which is a weak solution of (4.28) to obtain

$$
\left\|\nabla u_{M}\right\|_{L^{p}\left(\Omega_{R}\left(y_{0}\right), \omega\right)} \leq C\left[\left\|\mathbf{F}_{M} / \mu\right\|_{L^{p}\left(\Omega_{2 R}\left(y_{0}\right), \omega\right)}+\left\|g_{M}\right\|_{L^{p}\left(\Omega_{2 R}\left(y_{0}\right), \omega\right)}+\omega\left(\Omega_{R}\left(y_{0}\right)\right)^{1 / p}\right] .
$$

Then, by multiplying this equality with $M$, we obtain

$$
\|\nabla u\|_{L^{p}\left(\Omega_{R}\left(y_{0}\right), \omega\right)} \leq C\left[\|\mathbf{F} / \mu\|_{L^{p}\left(\Omega_{2 R}\left(y_{0}\right), \omega\right)}+\|g\|_{L^{p}\left(\Omega_{2 R}\left(y_{0}\right), \omega\right)}+\omega\left(\Omega_{R}\left(y_{0}\right)\right)^{1 / p} K_{0}\right] .
$$

The proof of Theorem 1.6 is therefore complete if we can prove (4.30). To this end, using the doubling property of $\omega \in A_{q}$, Lemma2.2, we have

$$
\frac{\omega\left(E_{M}\right)}{\omega\left(B_{r_{0}}(y)\right)}=\frac{\omega\left(E_{M}\right)}{\omega\left(B_{2 R}\left(y_{0}\right)\right)} \frac{\omega\left(B_{2 R}\left(y_{0}\right)\right)}{\omega\left(B_{r_{0}}(y)\right)} \leq M_{2} \frac{\omega\left(E_{M}\right)}{\omega\left(B_{2 R}\left(y_{0}\right)\right)}\left(\frac{2 R}{r_{0}}\right)^{n q} .
$$


From this, and using Lemma 2.2 again, we can find $\beta=\beta\left(M_{2}, n\right)>0$ such that

$$
\frac{\omega\left(E_{M}\right)}{\omega\left(B_{r_{0}}(y)\right)} \leq C\left(M_{0}, M_{2}, n\right)\left(\frac{2 R}{r_{0}}\right)^{n q}\left(\frac{\mu\left(E_{M}\right)}{\mu\left(B_{2 R}\left(y_{0}\right)\right)}\right)^{\beta / 2} \text {. }
$$

Now, by the definition of $E_{M}$, and the weak type (1-1) estimate for maximal function, we see that

$$
\begin{aligned}
\frac{\mu\left(E_{M}\right)}{\mu\left(B_{2 R}\left(y_{0}\right)\right)} & =\mu\left(\left\{\Omega_{R}\left(y_{0}\right): \mathcal{M}_{\mu, \Omega_{2 R}\left(y_{0}\right)}\left(|\nabla u|^{2}\right)>N M^{2}\right\}\right) \\
& \leq \frac{C\left(M_{0}, n\right)}{N M^{2}} \frac{1}{\mu\left(B_{2 R}\left(y_{0}\right)\right)} \int_{\Omega_{2 R}\left(y_{0}\right)}|\nabla u|^{2} \mu(x) d x \\
& \leq \frac{C\left(M_{0}, n\right) K_{0}^{2}}{N M^{2}},
\end{aligned}
$$

where $K_{0}$ is defined in 4.29). From this, and 4.31), it follows that

$$
\frac{\omega\left(E_{M}\right)}{\omega\left(B_{r_{0}}(y)\right)} \leq C^{*}\left(\Lambda, M_{0}, M_{2}, n\right)\left(\frac{2 R}{r_{0}}\right)^{n q}\left(\frac{K_{0}}{M}\right)^{\beta}
$$

Now, we choose $M$ such that

$$
M=K_{0}\left[\epsilon^{-1} C^{*}\left(\Lambda, M_{0}, M_{2}, n\right)\left(\frac{2 R}{r_{0}}\right)^{n q}\right]^{1 / \beta}
$$

then, it follows

$$
\omega\left(E_{M}\right) \leq \epsilon \omega\left(B_{r_{0}}(y)\right), \quad \forall y \in \bar{\Omega}_{R}\left(y_{0}\right) .
$$

This proves (4.30) and completes the proof.

\section{Appendix A. Proof of Lemma 2.7}

Proof. We follow the method used in [6, 27] with some modifications fitting to our setting. For each $x \in C$, let us define

$$
\phi(\rho)=\frac{\omega\left(C \cap B_{\rho}(x)\right)}{\omega\left(B_{\rho}(x)\right)} .
$$

By the Lebesgue Differentiation Theorem, for almost every $x \in C, \phi$ is continuous and $\phi(0)=\lim _{\rho \rightarrow 0^{+}} \phi(\rho)=1$. Moreover, by (i), $\phi\left(r_{0}\right)<\epsilon$. Therefore, for almost every $x \in C$, there is $0<\rho_{x}<r_{0}$ such that

$$
\begin{gathered}
\omega\left(C \cap B_{\rho_{x}}(x)\right)=\epsilon \omega\left(B_{\rho_{x}}(x)\right), \quad \text { and } \\
\omega\left(C \cap B_{\rho}(x)\right)<\epsilon \omega\left(B_{\rho}(x)\right), \quad \rho>\rho_{x} .
\end{gathered}
$$

Now, observe that the family of balls $\left\{B_{\rho_{x}}(x)\right\}_{x \in C}$ covers $C$. Therefore, by Vitali's Covering Lemma, there exists a countable $\left\{x_{k}\right\}_{k \in \mathbb{N}}$ in $C$ such that the balss $\left\{B_{\rho_{k}}\left(x_{k}\right)\right\}_{k \in \mathbb{N}}$ with $\rho_{k}=\rho_{x_{k}}$ are disjoint and

$$
C \subset \cup_{k=1}^{\infty} B_{5 \rho_{k}}\left(x_{k}\right) .
$$

From this, A.1 and Lemma 2.2, we infer that

$$
\begin{aligned}
\omega(C) & \leq \omega\left(C \cap\left(\cup_{k=1}^{\infty} B_{5 \rho_{k}}\left(x_{k}\right)\right)\right) \leq \sum_{k=1}^{N} \omega\left(C \cap B_{5 \rho_{k}}\left(x_{k}\right)\right) \\
& <\epsilon \sum_{k=1}^{N} \omega\left(B_{5 \rho_{k}}\left(x_{k}\right)\right) \leq \epsilon M 5^{n p} \sum_{k=1}^{N} \omega\left(B_{\rho_{k}}\left(x_{k}\right)\right) .
\end{aligned}
$$

Observe that by (i), and since $B_{\rho_{k}}\left(x_{k}\right)$ are all disjoint,

$$
\left(\cup_{k=1}^{n} B_{\rho_{k}}\left(x_{k}\right)\right) \cap \Omega_{R}\left(y_{0}\right) \subset D .
$$

We claim that

$$
\omega\left(B_{\rho}(x)\right) \leq \max M\left\{A^{-1}, 4^{n}\right\}^{q} \omega\left(B_{\rho}(x) \cap \Omega_{R}\left(y_{0}\right)\right), \quad \forall x \in \Omega_{R}\left(y_{0}\right), \rho \in\left(0, r_{0}\right) .
$$


From this claim, it follows that

$$
\omega(C) \leq \epsilon^{\prime} \sum_{k=1}^{n} \omega\left(B_{\rho_{k}}\left(x_{k}\right) \cap \Omega_{R}\left(y_{0}\right)\right)=\epsilon^{\prime} \omega\left(\left(\cup_{k=1}^{n} B_{\rho_{k}}\left(x_{k}\right)\right) \cap \Omega_{R}\left(y_{0}\right)\right) \leq \epsilon^{\prime} \omega(D) .
$$

It now remains to prove A.2. It follows from Lemma2.2 that

$$
\frac{\omega\left(B_{\rho}(x)\right)}{\omega\left(B_{\rho}(x) \cap \Omega_{R}\left(y_{0}\right)\right)} \leq M\left(\frac{\left|B_{\rho}(x)\right|}{\left|B_{\rho}(x) \cap \Omega_{R}\left(y_{0}\right)\right|}\right)^{q} .
$$

Hence, it suffices to prove

$$
\sup _{x \in \Omega_{R}\left(y_{0}\right)} \sup _{0<\rho<r_{0}} \frac{\left|B_{\rho}(x)\right|}{\left|B_{\rho}(x) \cap \Omega_{R}\left(y_{0}\right)\right|} \leq\left\{A^{-1}, 4^{n}\right\} .
$$

We fix $x \in \Omega_{R}\left(y_{0}\right)$ and $\rho \in\left(0, r_{0}\right)$. Observe that if $B_{\rho}(x) \subset \Omega_{R}\left(y_{0}\right)$. Then (A.3) is trivial. Hence, we only need to consider the case $B_{\rho}(x) \cap \partial \Omega_{R}\left(y_{0}\right) \neq \emptyset$. We divide this situation into three subcases.

Case I: If $B_{\rho}(x) \cap\left(\partial B_{R}\left(y_{0}\right) \cap \bar{\Omega}\right) \neq \emptyset$ and $B_{\rho}(x) \cap \partial \Omega \cap \bar{B}_{R}\left(y_{0}\right)=\emptyset$. Then, it follows that $\Omega_{R}\left(y_{0}\right) \cap B_{\rho}(x)=$ $B_{R}\left(y_{0}\right) \cap B_{\rho}(x)$. From this, a simple calculation shows

$$
\frac{\left|B_{\rho}(x)\right|}{\left|B_{\rho}(x) \cap \Omega_{R}\left(y_{0}\right)\right|}=\frac{\left|B_{\rho}(x)\right|}{\left|B_{\rho}(x) \cap B_{R}\left(y_{0}\right)\right|} \leq 4^{n} .
$$

Case II: If $B_{\rho}(x) \cap \partial \Omega \cap \bar{B}_{R}\left(y_{0}\right) \neq \emptyset$ and $B_{\rho}(x) \cap \partial B_{R}\left(y_{0}\right) \cap \bar{\Omega}=\emptyset$. In this case, by the proof of [27, Lemma 3.8], it follows that

$$
\frac{\left|B_{\rho}(x)\right|}{\left|B_{\rho}(x) \cap \Omega_{R}\left(y_{0}\right)\right|} \leq\left(\frac{2}{1-4 \delta}\right)^{n} \leq 4^{n} .
$$

Case III: If $B_{\rho}(x) \cap \partial \Omega \cap \bar{B}_{R}\left(y_{0}\right) \neq \emptyset$ and $B_{\rho}(x) \cap \partial B_{R}\left(y_{0}\right) \cap \bar{\Omega} \neq \emptyset$. Then, by the definition of type $\left(A, r_{0}\right)$ domain, we see that

$$
\left|\Omega_{R}\left(y_{0}\right) \cap B_{\rho}(x)\right| \geq A\left|B_{\rho}(x)\right|
$$

Therefore,

This completes the proof.

$$
\frac{\left|B_{\rho}(x)\right|}{\left|\Omega_{R}\left(y_{0}\right) \cap B_{\rho}(x)\right|} \leq A^{-1}
$$

Acknowledgement. T. Phan's research is supported by the Simons Foundation, grant \# 354889.

\section{REFERENCES}

[1] K. Adimurthi, T. Mengesha, and N. C. Phuc, Gradient weighted norm inequalities for linear elliptic equations with discontinuous coefficients, submitted.

[2] V. Bölegein, Global Calderón - Zygmund theory for nonlinear parabolic system, Calc. Var. (2014) 51:555 - 596.

[3] S. Byun, L. Wang, Nonlinear gradient estimates for elliptic equations of general type. Calc. Var. Partial Differ. Equ. 45(34), 403-419 (2012).

[4] S-S. Byun, Parabolic equations with BMO coefficients in Lipschitz domains. J. Differential Equations 209 (2005), no. 2, 229-265.

[5] S.-S. Byun, L. Wang, Parabolic equations in Reifenberg domains. Arch. Ration. Mech. Anal. 176 (2005), no. 2, $271-301$.

[6] S.-S. Byun, L. Wang, Elliptic equations with BMO coefficients in Reifenberg domains. Comm. Pure Appl. Math. 57 (2004), no. $10,1283-1310$.

[7] L.A. Caffarelli and I. Peral. On $W^{1, p}$ estimates for elliptic equations in divergence form. Comm. Pure Appl. Math. 51 (1998), no. $1,1-21$.

[8] D. Cao, T. Mengesha, T. Phan, Weighted $W^{1, p}$-estimates for weak solutions of degenerate and singular elliptic equations, 2016, arXiv:1612.05583 submitted.

[9] F. Chiarenza, M. Frasca, and P. Longo, Interior $W^{2, p}$ estimates for nondivergence elliptic equations with discontinuous coefficients, Ricerche Mat., 40 (1991), 149-168. 
[10] R. R. Coifman, C. Fefferman, Weighted norm inequalities for maximal functions and singular integrals. Studia Math. 51 (1974), 241-250.

[11] D. Cruz-Uribe, New proofs of two-weight norm inequalities for the maximal operator, Georgian Math. J. 7 (2000), no. 1, $33-42$.

[12] G. Di Fazio, M. A. Ragusa, Interior estimates in Morrey spaces for stong to non divergence form equations with discontinuous coefficients, J. of Fun. Ana., 112 (1993), 241- 256.

[13] G. Di Fazio, $L^{p}$ estimates for divergence form elliptic equations with discontinuous coefficients, Boll. Un. Mat. Ital., A 10 (1996).

[14] G. Di Fazio M. S. Fanciullo, P. Zamboni, Interior $L^{p}$ estimates for degenerate elliptic equations in divergence form with VMO coefficients. Differential Integral Equations 25 (2012), no. 7-8, 619-628.

[15] E. B. Fabes, C. E. Kenig, R. P. Serapioni, The local regularity of solutions of degenerate elliptic equations. Comm. Partial Differential Equations 7 (1982), no. 1, 77 - 116.

[16] J. Heinonen, T. Kilpeläinen, O. Martio, Nonlinear potential theory of degenerate elliptic equations. Dover Publications, Inc., Mineola, NY, 2006.

[17] L. T. Hoang, T. V. Nguyen, T. V. Phan, Gradient estimates and global existence of smooth solutions to a cross-diffusion system. SIAM J. Math. Anal. 47 (2015), no. 3, 2122-2177.

[18] R. A. Hunt, D. S. Kurtz, C. J. Neugebauer, A note on the equivalence of Ap and Sawyer's condition for equal weights. Conference on harmonic analysis in honor of Antoni Zygmund, Vol. I, II (Chicago, Ill., 1981), 156-158.

[19] J. Garcia-Cuerva, Weighted $H^{p}$-spaces, Thesis (Ph.D.)Washington University in St. Louis. 1975.

[20] J. Garcia-Cuerva, Weighted $H^{p}$ spaces. Dissertationes Math. (Rozprawy Mat.) 162 (1979).

[21] D. Gilbarg and N. Trudinger, Elliptic Partial Differential Equations of Second Order, Classics in Mathematics, SpringerVerlag, Berlin (2001).

[22] C. Gutiérrez, R. Wheden, Harnack's inequality for degenerate parabolic equations, Com. in Partial Differential Equations, 16 (4\&5), 1991, 745-770.

[23] D. Kim and N.V. Krylov, Elliptic differential equations with coefficients measurable with respect to one variable and VMO with respect to the others, SIAM J. Math. Anal., 39 (2007), 489-506.

[24] J. Kinnunen and S. Zhou, A local estimate for nonlinear equations with discontinuous coefficients, Comm. Partial Differential Equations 24 (1999), 2043-2068.

[25] N. V. Krylov, Lectures on elliptic and parabolic equations in Sobolev spaces. Graduate Studies in Mathematics, 96. American Mathematical Society, Providence, RI, 2008.

[26] A. Maugeri, D. K. Palagachev, L. G. Softova, Elliptic and parabolic equations with discontinuous coefficients. Mathematical Research, 109. Wiley-VCH Verlag Berlin GmbH, Berlin, 2000.

[27] T. Mengesha, N. C. Phuc, Weighted and regularity estimates for nonlinear equations on Reifenberg flat domains, J. of Diff. Eqns 250, 1, 1485-2507, 2011.

[28] T. Mengesha, N. C. Phuc, Global estimates for quasilinear elliptic equations on Reifenberg flat domains. Arch. Ration. Mech. Anal. 203, 189-216 (2011).

[29] N.G. Meyers. An $L^{p}$-estimate for the gradient of solutions of second order elliptic divergence equations. Ann. Sc. Norm. Super. Pisa Cl. Sci. (3) 17 (1963) 189 189-206.

[30] D. D. Monticelli, S. Rodney, R. L. Wheeden, Harnack's inequality and Hölder continuity for weak solutions of degenerate quasilinear equations with rough coefficients, Nonlinear Anal. 126 (2015), 69-114.

[31] D. D. Monticelli, S. Rodney, R. L. Wheeden, Boundedness of weak solutions of degenerate quasilinear equations with rough coefficients. Differential Integral Equations 25 (2012), no. 1-2, 143-200.

[32] B. Muckenhoupt and R. L. Wheeden. Weighted bounded mean oscillation and the Hilbert transform. Studia Mathematica, T. LIV. 1976.

[33] B. Muckenhoupt and R. L. Wheeden. On the dual of weighted $H^{1}$ of the half-space. Studia Mathematica, T. LXIII. 1978.

[34] B. Muckenhoupt, Weighted norm inequalities for the Hardy maximal function. Trans. Amer. Math. Soc. 165 (1972), $207-226$.

[35] M. K. V. Murthy, G. Stampacchia, Boundary value problems for some degenerate-elliptic operators. Ann. Mat. Pura Appl. (4) $80,1968,1-122$.

[36] T. Nguyen, Interior Calderón-Zygmund estimates for solutions to general parabolic equations of p-Laplacian type, 2016 , submitted.

[37] T. Nguyen, T. Phan, Interior gradient estimates for quasilinear elliptic equations, Calc. Var. Partial Differ. Equ., DOI: 10.1007/s00526-016-0996-5.

[38] K. Nyström, H. Persson, and O. Sande, Boundary estimates for solutions to linear degenerate parabolic equations. J. Differential Equations 259 (2015), no. 8, 3577-3614.

[39] E. Sawyer, A characterization of a two weighted norm inequality for maximal operators, Studia Mathematica, T. LXXV (1982).

[40] E. M. Stein, Harmonic analysis: real-variable methods, orthogonality, and oscillatory integrals. Princeton Mathematical Series, 43. Monographs in Harmonic Analysis, III. Princeton University Press, Princeton, N.J., 1993. 
[41] M. Surnachev, A Harnack inequality for weighted degenerate parabolic equations. J. Differential Equations 248 (2010), no. 8, 2092-2129.

[42] E. Stredulinsky, Weighted inequalities and applications to degenerate elliptic Partial Differential Eequations Ph. D. Thesis, Indiana University, 1981.

[43] I. E. Verbitsky, Weighted norm inequalities for maximal operators and Pisier's theorem on factorization through $L^{p, \infty}$. Integral Equations Operator Theory 15 (1992), no. 1, 124-153.

[44] L. Wang, A geometric approach to the Calderón-Zygmund estimates, Acta. Math. Sin. (Engl. Ser.), 19 (2003), $381-396$.

Department of Mathematics, University of Tennessee, Knoxville, 227 Ayres Hall, 1403 Circle Drive, Knoxville, TN 37996, U.S.A.

E-mail address: phan@math.utk.edu 